\title{
The Logic of Disorder: A Dynamic View of Cognitive Aesthetics
}

by

\author{
SAMANTHA SCHARTMAN \\ Submitted in partial fulfillment of the requirements \\ For the degree of Master of Arts
}

Thesis Advisor: Dr. Per Aage Brandt

Case Western Reserve University

Department of Cognitive Science

May 2010 


\section{CASE WESTERN RESERVE UNIVERSITY \\ SCHOOL OF GRADUATE STUDIES}

We hereby approve the thesis/dissertation of
Samantha Schartman
candidate for the $\quad$ Master of Arts $\quad$ Per Aage Brandt $*$
(chair of the committee)
Todd Oakley

(date) $\quad$ March $26^{\text {th }} 2010$

*We also certify that written approval has been obtained for any proprietary material contained therein. 


\section{Table of Contents}

II. INDEX OF FIGURES 2

III. ACKNOWLEDGEMENTS 4

IV. ABSTRACT $\quad 5$

V. BEGINNINGS: INTRODUCING RUDOLF ARNHEIM 6

VI. ENTROPY: PHYSICS FOR ARTISTS

VII. CONVERGENCE: ARNHEIM AND ENTROPY 9

VIII. ENTROPY AND ART: AN ARTICLE EXPLAINED 12

IX. STATING THE MISSION: AN INTRODUCTION TO METHOD 18

X. OPPOSITION ALONG A CONTINUUM: BAUDELAIRE $\quad 19$

XI. THEMATIC OPPOSITION IN BAUDELAIRE'S LES CHATS 21

XII. NARRATIVE DYNAMICS IN BALZAC

XIII. FRAMING THE ONTOLOGY: REFLEXIVITY AND COGNITIVE SEMIOTIC NETWORKS32

XIV. AN ACTANTIAL MODEL OF MONET'S ROUEN CATHEDRAL

XV. GREIMAS AND THE SEMIOTIC SQUARE $\quad 42$

XVI. EMBODIED DUALITIES: WALLACE STEVENS $\quad 45$

XVII. COGNITIVE SEMIOTIC BLENDING: A CLOSER LOOK AT WALLACE STEVENS 51

XVIII. SEMIOTIC BLENDING AND SIMILES: LOREN EISELEY

XIX. REVERSIBILITY VS. IRREVERSIBILITY: A DISCUSSION OF TEMPORALITY IN ART 62

\begin{tabular}{lr} 
XX. DIEGESIS: CY TWOMBLY & 62 \\
\hline
\end{tabular}

XXI. CONCLUSION

XXII. IMPLICATIONS FOR FURTHER RESEARCH

\begin{tabular}{lr} 
XXIII. BIBLIOGRAPHY & 79 \\
\hline
\end{tabular} 


\section{Index of Figures}

Figure 1 - reprinted diagram found on p. 19 (corresponds to figure 3) of Rudolf Arnheim's Entropy and Art (1971)

Figure 2 - this diagram represent my best attempt to map the theory set forth by Arnheim. It would appear to me that he intends to describe the process that Mircea Eliade called the "eternal return" This would in fact correspond to the $2^{\text {nd }}$ law of thermodynamics in that it supports that energy is neither created nor destroyed, but rather transformed. If I am correct in my understanding of Arnheim's theory, his intertwining terminology functions to describe different steps in a larger process of dynamic becoming. This is not to imply though, that the cycle seamlessly moves in a single direction continuously, on the contrary! A dynamic element might move toward and then away from any of the stages at any given time. 18

Figure 3 - Diagram of Baudelaire's "beauty" continuum............................................... 20 Figure 4 - Diagram of themes parallel to the semantic networks Proximal and Distal

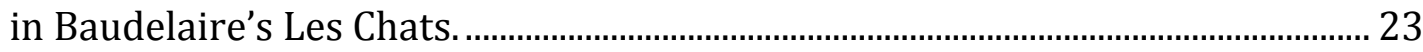

Figure 5 - The underlying force-dynamic theme represented by the character "Porbus" throughout the text....................................................................................... 26

Figure 6 - The underlying force-dynamic theme represented by the character

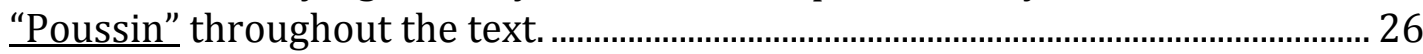

Figure 7 - The underlying force-dynamic theme represented by the character

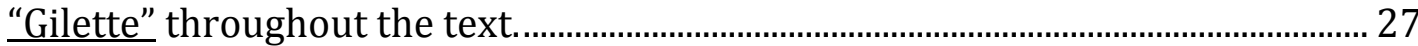

Figure 8 - The underlying force-dynamic theme represented by the character "Frenhofer's Masterpiece" throughout the text.

Figure 9 - in this mapping, the "masterpiece" is referred to in two separate parts "a" and " $b$ " corresponding to the states of the painting described in the text. In the beginning, the "masterpiece" is being brought closer to "perfection", ("a") during this time; Frenhofer is seen as a genius creating a

Figure 10 - This mapping had the same meaning as the one preceding, just constructed another way. The main difference between these two methods of mapping is in how the continuum is illustrated. In the preceding mapping, the continuum is shown as a gradient between two end-points, in this one, it is inferred that the continuum goes on forever, oscillating between ordered and disordered states of being.

Figure 11 - Favoring, for the moment, the mapping style described in figure 7, this mapping illustrates the combined force dynamic oppositions present in the text.

Figure 12 - In the style of figure 8, this mapping illustrates the overarching theme of the narrative structure.

Figure 13 - this is a recreation of the ontological graph that appears in Dr. Per Aage Brandt's working paper titled "The world. A view from within"..............................32

Figure 14 - Claude Monet's Rouen Cathedral ................................................................... 35 
Figure 15 - generic actantial narrative model describing the placement of variables.

Figure 16 - Actantial Narrative Model of Gombrich's view of Monet's approach to

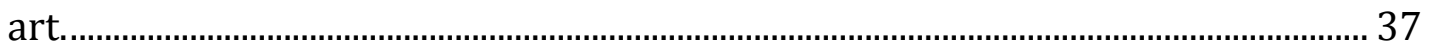

Figure 17 - Actantial Narrative Model of Monet's approach to art ................................ 38

Figure 18 - Semiotic blending network illustrating the duality of art as both signifier and signified with our reactions to it as a contributor to culture........................... 40

Figure 19 - Logical Negation in Greimas' semiotic square............................................... 43

Figure 20 - Dynamic version of the semiotic square model described by Greimas-

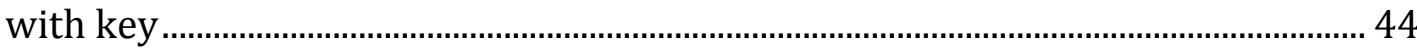

Figure 21 - Greimas' Semiotic Square depicting the dynamic structure of Steven's poem "The Poems Of Our Climate"............................................................................ 49

Figure 22 - Proposed semiotic blending cascade for "The Poems Of Our Climate" .. 52 Figure 23 - Semiotic Blending Cascade for the Loren Eiseley poem "Notes of an Alchemist" 58

Figure 24 - Semiotic square describing the relevance-making schema in the phenomenological layer of the mapping of Loren Eiseley's poem "Notes of an Alchemist"

Figure 25 - Semiotic square describing the relevance-making schema in the semiotic layer of the mapping of Loren Eiseley's poem "Notes of an Alchemist" ............... 61

Figure 26 - Work on paper by Cy Twombly entitled "Virgil" (oil, chalk, and crayon on paper). Private collection, Berlin.

Figure 27 - The (dynamic) semiotic square (grey) extended by the (static) Brandtian Diegesis model (green) indicating the "surplus" of the intentional and showing how an amplification of one of the opposing forces can result in the discontinuation of the dynamic found in the semiotic square. 64

Figure 28 - Semiotic Square and Diegesis Model of Cy Twombly's work entitled "Virgil". 65

Figure 29 - semiotic square model of music 69

Figure 30 - Score for Cornelius Cardew's The Great Learning from 1971, taken from paragraph 7 of the Confucian text of the same name. Text translated into English by Ezra Pound.................................................................................................. 70

Figure 31 - Semiotic Square and Diegesis Model of the musical piece by Cornelius Cardew's entitled Paragraph 7. 


\section{Acknowledgements}

To: Charles Bergengren, Per Aage Brandt, Todd Oakley, Saul Ostrow, and Mark Turner Also: John Cycyk, and Deborah Schartman 


\title{
IV. Abstract
}

\section{The Logic of Disorder: a Dynamic View of Cognitive Aesthetics}

\author{
Abstract
}

by

\section{SAMANTHA SCHARTMAN}

In observing various patterns of organization I have come to a similar conclusion as that of seminal theorist and psychologist Rudolf Arnheim; that there is a neg-entropic drive towards order as exemplified by the presence of phenomena such as societies, governments and grammars. In contrast to this, due to the fact that we are embodied beings, there is also an entropic drive (germinating from human phenomenology) towards chaos. We see this demonstrated by the existence of emotion, free will, weather patterns and so on. These conflicting drives follow along an order/disorder axis. As these drives follow along this continuum in opposite directions, a tension, or force dynamic relationship, is created between the entropic and the neg-entropic.

I assert that it is this tension that results in aesthetic appeal or dynamism and that this effect, which is not specific to any one modality, is a discrete character of the cognitive underpinnings of the aesthetic experience. 


\section{Beginnings: Introducing Rudolf Arnheim}

"All perceiving is also thinking, all reasoning is also intuition, all observation is also invention" ${ }^{1 "}$

- Rudolf Arnheim

Rudolf Arnheim, now recognized as a distinguished psychologist and art theorist, was born in Berlin in 1904 and enrolled at the University of Berlin in 1923 where he studied with two of the founders of Gestalt psychology; Max Wertheimer and Wolfgang Köhler. Until his passing in 2007 at the age of 102, Rudolf Arnheim continued to write, research and engage in the field of psychology with the aim to "narrow the gap between scientific and artistic knowledge" (Behrens, 1). In his article, Entropy and Art written in 1971, Arnheim "reflected on the requirements for macroscopic/perceived order in the context of the theory of physical systems"(Verstegen, 7).

Drawing structurally from the $2^{\text {nd }}$ Law of Thermodynamics, Arnheim asserted that there are both downward "catabolic" (entropy) forces and also upward "anabolic" (neg-entropy) forces in physical systems resulting in a dynamic equilibrium of elements. This dynamism, which is manifested both perceptually (visibly) $^{2}$ and structurally (imperceptibly), is said by Arnheim to reflect the order of the physical world in that "outer order so often represents inner functional order.(Arnheim, 2)".

\footnotetext{
${ }^{1}$ Rudolf Arnheim, Art and Visual Perception: A Psychology of the Creative Eye: The New Version (University of California Press, 1974) p. 5

2 One can observe the appearance of order and disorder in any given thing.
} 
Arnheim's interest in this line of inquiry springs from his desire to reconcile human creativity and art with the scientifically measurable systems found in the domains of science and biology. Assuming that human beings are creative, and can be biologically studied, and that human cognition can be approached as an emergent order represented by our internal biological/functional order, Arnheim approaches Art as an analyzable product of the intentional union of humankind's cognition and physical being.

It is this theory that serves as the originating catalyst for the present inquiry that endeavors to analyze multiple modes of aesthetic communication in order to reveal and define the force dynamic pattern of order and disorder that arises from our embodied nature as author and audience in pursuit of the perceptually ideal (aesthetically dynamic) form. By analyzing how the play of entropy and negentropy function across multiple instantiations of creativity, I aim to uncover some baseline force dynamic consistency that implicates a unifying property of art. Through this research into the underpinnings of the aesthetic experience I aim to present and define a general principle of cognitive aesthetics.

\section{Entropy: Physics for Artists}

"Just as the constant increase of entropy is the basic law of the universe, so it is the basic law of life to be ever more highly structured and to struggle against entropy. - Vaclav Havel 
Entropy, as understood in the context of physics and the $2^{\text {nd }}$ law of thermodynamics, states that in any closed system, there exists a tendency towards the dissipation of useful energy. ${ }^{3}$ Describing one function of a closed system as asserting a directed, "intentional" drive towards the production of work through the constriction of a (mechanistic) framework (i.e. system boundary), and considering the effects of more or less efficient constraints, "entropy was eventually redefined statistically in terms of "order" and "disorder"'4 (Freund, 495). While the $2^{\text {nd }}$ Law of thermodynamics, which asserts that entropy increases until equilibrium ${ }^{5}$, specifically relates to closed systems; the world, with human beings intertwined, cannot be defined only as ever-increasing entropy nor can it be considered a purely closed system at the human scale. ${ }^{6}$ In applying the notion of entropy to an open system, while one must retain the significance of the term as a tendency towards disorder, the part of the law stating that entropy can never decrease (which only pertains to closed systems) becomes void; thus allowing both entropic and negentropic drives to be present in the same system.

\footnotetext{
3 The notion of entropy is in no way fully explored in the present article, nor should it be, as the complexity of its definition can be approached from a number of fields. What I assert here is that the foundational understanding of Entropy as a dissipation of energy, or drive towards disorder (as corresponds with time's arrow) represents a useful basis for the creation of a functional metaphorical model, and that this model could, through additional exploration and analysis, provide useful implications for further research.

${ }^{4}$ When the molecular theory of heat replaced the caloric theory, entropy was eventually redefined statistically in terms of "order" and "disorder." (Freund, 495)

5 The second law of thermodynamics demands that the entropy of an isolated physical system will increase (Stern, 227).

${ }^{6}$ Mark Turner often discusses the ability of human beings to compress expansive ideas to a relatable scale. Here I do just this as our "world" could indeed be considered, on a cosmic scale, a "closed system" with the cosmos denoting the system's boundary. However, pertinent to the current conversation is the maintenance and acknowledgment of the perspective of human consciousness.
} 
One salient example of an open system is the art object; while it is possible to think of an art object as a closed system subject to the effects of time manifested by physical deterioration, it is more significantly defined as an open system; which takes into account a function of art as the culturally inclusive and unresolved foci for dialogue and the exchange of ideas. From these examples, the notion of a closed system can be defined as a temporary network, where no new information, energy or input can be added. And open system, on the other hand, is continually and dynamically altered by outside elements. This perspective, (and corresponding definition of entropy) which can be applied not only to the art object, but also to other aesthetic phenomena such as music, literature, performance, dance, and film, will be the basis for the subsequent inquiry.

\section{Convergence: Arnheim and Entropy}

"Order is a necessary condition for anything the human mind is to understand." - Rudolf Arnheim

Rudolf Arnheim was not always interested in entropy. His seminal book Art and Visual Perception centered on the methods and processes we undergo as human beings, both consciously and subconsciously, when we experience art. But for all his insight into the ways we perceive and understand things like shape, space, light, color, and movement, the bulk of his book did not address the almighty notion of beauty; what it is about the combination of these things that inspires in us, if only 
for a fleeting moment, a heightened connection with life. It was only in his second to last chapter entitled Dynamics that he began his search for a general theory that would define the "activation" of his theoretical gestalt. This search resulted in his article Entropy and Art (1971) (and a revision of his book in 1974) in which he sought to lay out the structure by which the $2^{\text {nd }}$ law of thermodynamics corresponded to the way macroscopic elements were created and perceived aesthetically. This attempt at building a bridge between science and the humanities was an early attempt at considering the cognitive/neurological processes underlying the creation and perception of aesthetic phenomena. In his own words:

If there were independent evidence to make it likely that a similar tendency towards orderly structure exists in these brain processes also, one might want to think of perceptual order as the conscious manifestation of a more universal physiological and indeed physical phenomenon (Arnheim, 3).

As explained earlier, Entropy, in a simplified sense, is the dissipation of energy that is available to do work in a closed system; in other words, it is a reduction of ordered/directed/focused productivity, a tendency towards disorder. But while the $2^{\text {nd }}$ Law asserts that energy only moves from a state of order to one of disorder ${ }^{7}$, Arnheim observed that at the human scale (an open system), order is created out of chaos and homogeneity all the time; that the introduction of cognition/intelligence by human beings, into a system, strengthens (and often creates) the tendency towards order. It is here that I respond to Peter Landsberg, an established physicist who states:

\footnotetext{
${ }^{7}$ It is this law that serves to explain the phenomena of time and aging.
} 
A bridge from science to literature may be accomplished via science fiction. As you might expect from metaphors, the use of the entropy concept here is only suggestive and often superficial. Its normal use is as a hint at ultimate chaos and at a general tendency to decay, be it of objects, people or social organizations (Landsberg, 90).

First, I do not believe the present query to be rooted in science fiction; to make such a statement is to argue for a refutation of the field of cognitive science, which is one that, among many things, attempts to reconcile biological/neurological functions with that of our cognitive/perceptual experience. Second, the use of entropy theory, as a systemic universal pattern (an instance of metaphorical use as asserted by Landsberg) is far from merely suggestive and superficial, but rather attempts to explore the age-old claim "as above, so below"8. Arnheim concurs:

The perceivable order tends to be manifested and understood as a reflection of an underlying order, whether physical, social, or cognitive (Arnheim,2).

Last, using entropy theory to examine closed systems will, in fact, lead one to nihilistic conclusions of ultimate decay, but applying the framework to open systems such as human cognition, perception and experience, disposes one to much more productive $\mathrm{e}^{9}$ analogues that allow for the exchange of forces moving towards and away from disorder. An open system, as it relates to the current study, is defined as a continually dynamic network that evolves over time. This dynamism is characterized as an unremitting flow of input from the perceiver's temporal reality

\footnotetext{
8 This phrase comes from the beginning of The Emerald Tablet written by Hermes Trismegistus c.a. 1140 meaning that the Macrocosm is the same as microcosm.

${ }^{9}$ In artistic productivity, or creativity, the dynamics in question has become conscious and even intentional, as a sort of "technique", but in $20^{\text {th }}$ century thinking, the reduction of the intentional to causal processes was common and even fashionable (i.e. Structuralism).
} 
to their relevance schema or personal world perspective as it relates to a given experience. In contrast, a closed system is one in which the dynamism of a given network is temporary and nonreversible in its occurrence. This distinction will be further explained in later sections of this study.

Considering this, the use of Entropy theory, as applied to aesthetics, is more than a metaphor - a figure of speech applied to an object or action to which is not literally applicable-it represents an observed pattern that exists and is repeated throughout both the microcosm and macrocosm. This pattern is as relevant to cognitive scientists and psychologists as it is to physicists in regards to the productivity of the theory and its potential to unite cosmic principals with biological, and cognitive ones.

\section{Entropy and Art: An Article Explained}

Rudolf Arnheim's article Entropy and Art: an Essay on Disorder and Order (1971) is a daunting article for the layman; laced with passages of technical references to physics, thermodynamics (a highly debated area of study in and of itself), and information theory it is not surprising that 1.) the article spirited much discussion, and 2.) the theory was not significantly developed further. As a result of much analysis and fact checking with physicist friends, I have come to the conclusion that the reason for the lack of traction lies in Arnheim's contradictory statements and logic. 
While Arnheim's observations and justification for this research are not in question, and in fact are supported by the present article, as you will see, the logic of his system is lacking.

Focusing first on Arnheim's notion of "order", we need only read to the second page of his article:

But it is hard, perhaps impossible to find examples in which the order of a given object or event is limited to what is directly apparent in perception.

While this sounds perfectly logical, Arnheim fails to address these other, nonperceptible levels of order or disorder anywhere else in his article. Next we look for his notion of disorder:

Disorder is not the absence of all order but rather the clash of uncoordinated orders (Arnheim,7).

This definition of disorder seems problematic in that the "clash" he refers to is never given parameters, or a structured definition. It is as if he is inserting a qualitative value judgment in the systemic functioning of his network; who decides if the orders are at odds? By what set of criteria is a set order evaluated? The system he proposes lacks sufficient specificity, which in turn makes it difficult if not impossible to make the theory productive for others. The soup is already muddled.

Next, while Arnheim claims to be borrowing from the theory of entropy, he inserts two new concepts (which he attributes to himself): the catabolic effect, and the anabolic tendency. Arnheim defines the catabolic effect as: 
Such constraining partitions can be removed by the hand of man, but also by all sorts of natural violence, by crumbling and rusting, erosion or friction. I will call this destruction of shape the catabolic effect (Arnheim,15).

With no differentiation provided, it appears that the catabolic effect is akin to the effects of entropy, but what function, we might ask, does the shift in terminology have? While no direct answer is given, we are provided with the following diagram seen below in figure 1:

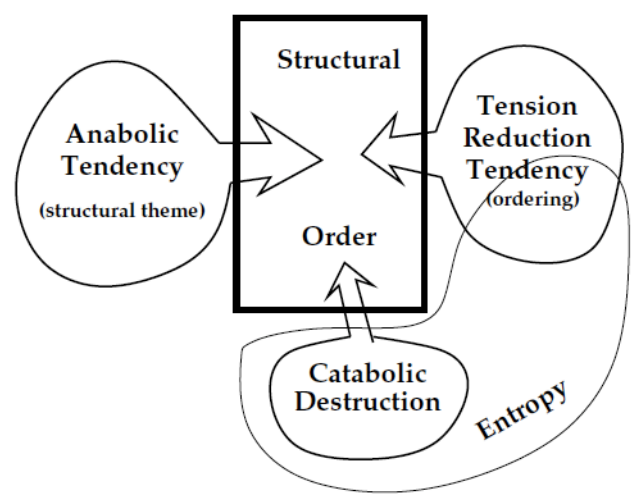

Figure 1 - reprinted diagram found on p. 19 (corresponds to figure 3) of Rudolf Arnheim's Entropy and Art (1971)

As we can see from the diagram, Arnheim intends this "catabolic effect" as some process of the greater notion of entropy; how this relationship is defined, is not clearly communicated. The only further qualification given is that this catabolic effect is "shape destroying" (which seems to indicate that catabolism is a process) and can be applied either directly through the intentional destruction of pattern, or indirectly through the removal of structurally defining constraints ${ }^{10}$. Perhaps

\footnotetext{
${ }^{10}$ Structural defining constraints seem to better correspond to a closed system in which constraints more directly add structure to form in a fixed as opposed to dynamic way.
} 
Arnheim's view of entropy will offer a more informative basis for our understanding?

The processes measured by the principle of entropy are perceived as the gradual or sudden destruction of inviolate objects-a degradation involving the breaking-up of shape, the dissolution of functional contexts, the abolition of meaningful location (Arnheim, 7).

From the above quotation, we can now begin to understand that the "catabolic effect" is the end result of the process of "catabolism", which is Arnheim's terminology for referring to the "Entropy principle" in time. In other words, catabolism is the formal process of the principle of Entropy.

Then, on Page 10, Arnheim add this definition:

Entropy theory, on the other hand, is not concerned with the probability of succession in a series of items but with the overall distribution of kinds of items in a given arrangement (Arnheim, 10).

Again, we have competing definitions: one that says that entropy deals with the erosion of shapes (Arnheim, 7), and one that says that it deals with the distribution of multiple items (Arnheim, 10). The implications set forth by each of these definitions presents a different picture, namely one that is concerned with form, and another that is interested in probability and the arrangement of multiples. Perhaps we need to refer back to his earlier assertion of how order is represented at many levels - both perceivable and unperceivable? But then it would seem that each of these definitions are more directed towards a particular scale as opposed to signifying a more universal concept. Needless to say, more clarity is needed. 
Turning now to Arnheim's definition of the anabolic tendency, we see again, the lack of distinguishing between it and the concept of neg-entropy. Neg-entropy, is understood as the inverse of entropy - the increase of energy within a system available to do work ${ }^{11}$. While it is not explicitly stated in Arnheim's article, building on what we have come to understand about the relationship between the principles of Entropy and Neg-entropy, I believe it is safe to assume that once again the introduction of the term, "Anabolism" (anabolic tendency) is used to differentiate between "entropy" as a cosmic principle and the perceptual process of its instantiation in time.

What again becomes confusing is that Arnheim later goes on to state that the anabolic tendency doesn't create without the interaction of tension reduction.

The anabolic tendency contributes what I will call the structural theme of a pattern, and this theme creates orderly form through interaction with the tendency to tension reduction (Arnheim, 17).

So what is tension reduction? This is the most difficult of all of the terms Arnheim puts forth. Let us first look at what the term "tension" means by itself, in relation to our larger concept of entropy:

However, what entropy theory measures is not the nature of organization ${ }^{12}$ but only its overall product, namely, the degree of dissipation of energy it entails, the amount of "tension" available for work in the system (Arnheim 12).

\footnotetext{
11 This rarely if ever happens within a closed system, per the $2^{\text {nd }}$ law of thermodynamics, so within the world of physics, the concept is automatically conceived of as taking pace within an open system. This suite our current inquiry just fine as our context, being human cognition and culture, is inherently an open system.

12 Interesting, considering Arnheim states that entropy is interested in the distribution of kinds of things in a given arrangement, does this not imply organization?
} 
It would seem that Arnheim uses the term "tension" to refer to energy within a given system, so this is the understanding I move forward with.

Considering the definition of "tension" as energy within a system, the compounded term "tension reduction" never directly defined, can only be assumed to be the dissipation/reduction of energy within a system. This sounds (dangerously) close to entropy does it not? But Arnheim defines it differently than a mere drive towards disorder; he describes it as a shift from an ordered state to a state of equilibrium (which he classifies as a low level order) (Arnheim, 18). The problem here again is that there is no criteria by which an entropic drive is differentiated from tension reduction; nor is there any fine-grained description of the relationship between tension reduction and the anabolic effect (neg-entropy in process). Once again we are left to draw our own conclusions. It is my understanding that tension reduction is, like the anabolic tendency, a structural theme, however is informed by the context in which the dynamics of anabolism and catabolism interact. This context offers the potential for other principles to stabilize the system resulting in a particular pattern. ${ }^{13}$

My best summation is that there is a basically good theory at work here underneath a lot of dysfunctional terminology. To risk an interpretation, it would seem that Arnheim is basically suggesting a circular concept of eternal becoming; something that might look a bit like this (Figure 2):

\footnotetext{
13 What is meant by "other principles" is the acknowledgement that there are more theories in the cosmos that might be relevant than just the theory of entropy and the $2^{\text {nd }}$ law of thermodynamics. Perhaps an example of an "other principle" is the theory of gravity, or that of planetary motion?
} 


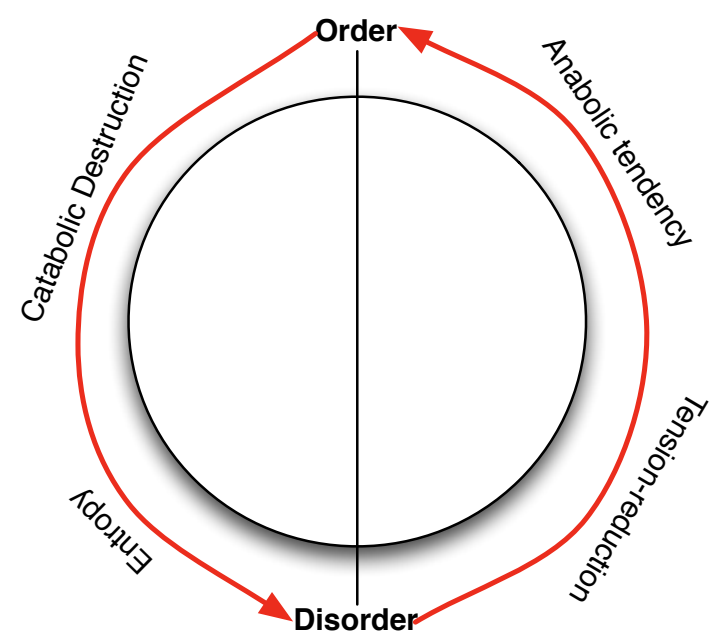

Figure 2 - this diagram represent my best attempt to map the theory set forth by Arnheim. It would appear to me that he intends to describe the process that Mircea Eliade called the "eternal return"14 This would in fact correspond to the $2^{\text {nd }}$ law of thermodynamics in that it supports that energy is neither created nor destroyed, but rather transformed. If I am correct in my understanding of Arnheim's theory, his intertwining terminology functions to describe different steps in a larger process of dynamic becoming. This is not to imply though, that the cycle seamlessly moves in a single direction continuously, on the contrary! A dynamic element might move toward and then away from any of the stages at any given time.

\section{Stating the mission: An Introduction to method}

The methodology set forth by Arnheim not withstanding, I endeavor to demonstrate an alternative approach that will borrow from Arnheim's observations and justifications for such a course of study. Using a more simplified vocabulary consisting of: disorder and order as static states along a continuum running from "perfect" order to "complete" chaos, and entropy and neg-entropy as the drives towards and away from disorder and order respectively, the present study will proceed towards a less confounding exploration of the universally observed pattern

\footnotetext{
14 Coincidentally, Mircea Eliade, acclaimed religious historian, coined the concept "the eternal return" in his book aptly named "The Myth of The Eternal Return: Cosmos and History" in the same year that Arnheim wrote his article "Entropy and Art": 1971.
} 
of order and disorder. In order to do so, I will employ many methodologies from Cognitive Science to look more closely at the elements at work within the semiotic systems being reviewed. In the following chapters I will outline and demonstrate these approaches to analysis by building on simple observed patterns and/or themes from which I will expand to more complex methods of analysis. In doing so, you will see, through the examples used to explain these methods, that the present assertion (that the effect of force dynamic tension represents a discrete character of the underpinnings of the aesthetic experience) is upheld consistently throughout each exploration, creating a solid foundation from which the implication of a unifying principle of cognitive aesthetics can be drawn.

\section{Opposition Along a Continuum: Baudelaire}

In 1855, poet and art critic Charles Baudelaire wrote a three-part review of the art being shown in the Exposition Universelle in Paris (Harrison, et al., 1998). In the first part of his review, Baudelaire makes a statement that I contend is indicative of a consistency between his approach to aesthetics and that which I assert in the present article.

"The beautiful is always strange. I do not mean that it is coldly, deliberately strange. For in that case, it would be a monstrosity that had jumped the rails of life. I mean that it always contains a touch of strangeness, and that it is this touch of strangeness that gives it its particular quality as beauty."

- Charles Baudelaire, 1855

In this statement, a duality is put forth through the fragment "always contains" which implicates both a "container" and a "contained". Adding to this, our 
understanding of "strangeness" as something unfamiliar, unsettling, or hard to understand, and the later reference to deliberate strangeness as something that runs contrary to life, we can imagine the following continuum along which beauty occurs:

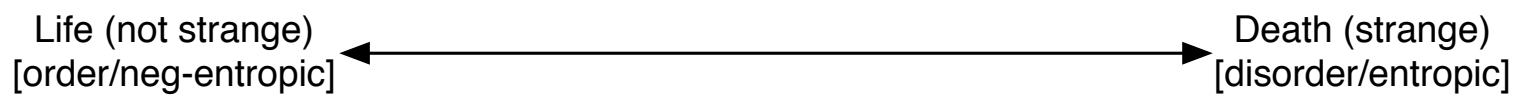

Figure 3 - Diagram of Baudelaire's "beauty" continuum

Now that we can visualize Baudelaire's continuum, we must consider how to understand it.

Returning to his phrase "always contains", we consider the function of the extremes of the scale represented. At one end, we have life, characterized by a tendency towards order (neg-entropic) in that cognitive processes and patterns of organization can be observed to be a result of human tendencies, and considered familiar or not strange. On the other end of the continuum, we have death - a state completely unfamiliar and enigmatic to us. We characterize movement towards death as deterioration of the familiar or a tendency towards disorder (entropy). Things that Baudelaire states are intentionally strange, or cold, have "jumped the rails of life", which means that they no longer are striving towards the ordered state of life. If we characterize beauty as that which "always contains" a touch of the strange, we can thus rule out the location of beauty (along our continuum) as being at either defined extreme. This leaves us with a schema that is consistent with 
present assertions that define aesthetic dynamism (beauty) as being a product of a force dynamic opposition between order and disorder.

\section{Thematic Opposition in Baudelaire's Les Chats}

Let us now test Baudelaire's aesthetic approach on his famous poem Les Chats from his seminal work: Fleurs du mal (1857).

\begin{tabular}{|c|c|}
\hline Les Chats & Cats \\
\hline $\begin{array}{l}\text { Les amoureux fervents et les savants } \\
\text { austères Aiment également, dans leur mûre } \\
\text { saison, Les chats puissants et doux, orgueil de la } \\
\text { maison, Qui comme eux sont frileux et comme } \\
\text { eux sédentaires. } \\
\text { Amis de la science et de la volupté Ils cherchent } \\
\text { le silence et l'horreur des ténèbres; L'Erèbe les } \\
\text { eût pris pour ses coursiers funèbres, S'ils } \\
\text { pouvaient au servage incliner leur fierté. } \\
\text { Ils prennent en songeant les nobles } \\
\text { attitudes Des grands sphinx allongés au fond des } \\
\text { solitudes, Qui semblent s'endormir dans un rêve } \\
\text { sans fin; } \\
\text { Leurs reins féconds sont pleins d'étincelles } \\
\text { magiques, Et des parcelles d'or, ainsi qu'un } \\
\text { sable fin, Etoilent vaguement leurs prunelles } \\
\text { mystiques. } \\
\text { - Charles Baudelaire }\end{array}$ & $\begin{array}{l}\text { Both ardent lovers and austere scholars Love in } \\
\text { their mature years The strong and gentle cats, } \\
\text { pride of the house, Who like them are sedentary } \\
\text { and sensitive to cold. } \\
\text { Friends of learning and sensual pleasure, They } \\
\text { seek the silence and the horror of darkness; } \\
\text { Erebus would have used them as his gloomy } \\
\text { steeds: If their pride could let them stoop to } \\
\text { bondage. } \\
\text { When they dream, they assume the noble } \\
\text { attitudes Of the mighty sphinxes stretched out } \\
\text { in solitude, Who seem to fall into a sleep of } \\
\text { endless dreams; } \\
\text { Their fertile loins are full of magic sparks, And } \\
\text { particles of gold, like fine grains of sand, } \\
\text { Spangle dimly their mystic eyes. } \\
\text { - William Aggeler, The Flowers of Evil (Fresno, CA: } \\
\text { Academy Library Guild, 1954) }\end{array}$ \\
\hline
\end{tabular}

*Original and translated version reproduced for reference.

In the first stanza of Baudelaire's Les Chats, the poet compares cats to both lovers (amoureux) and scholars (les savants) and adds that they are both strong (puissants) and gentle (doux); qualities that can be considered as corresponding to the before mentioned character-types. The emerging theme that arises at this early 
point in the poem relates to our observed force-dynamic pattern in that the notion of strength is related to asserted/intentional effort, in other words - an ordered state. On the other hand, the notion of gentility is associated with a mild temperament, or placid condition lacking asserted intentionality - a disordered state. So far our theme is in tact. Let us now explore the themes present in the second stanza.

In the second stanza, the themes of learning and sensual pleasure are introduced and then followed by the related themes of silence and darkness (one who studies needs a quiet environment to be with her thoughts, and darkness usually accompanies uninhibited moments for shame). While the secondary themes of silence and darkness are both associated with a disordered, lifeless state, the major themes of learning and sensual pleasure set up a different relationship; one that is characterized by the spiritual vs. carnal, the proximal vs. the distal. We will return to this important observation later.

Considering again the reoccurring theme of order and disorder, the end of the second stanza establishes the opposition of independence (intentional disorder) and servitude (intentional order) through the reference to Erebus (personification of darkness and the underworld who uses horses as transportation/messengers to and from the "world beyond")

Looking now to the third stanza, we see the repetition of the semantic networks relating to the proximal and the distal. This is evidenced in the reference to a cat's dreaming/thinking (en songeant) (proximal) and their likeness to the distal motif: the Sphinx. This pattern is repeated again through the reference to 
sleep as a proximal (familiar) occurrence and it's unfamiliar counterpart - infinity (endless dreams) (distal).

In the last stanza, Baudelaire asserts his affinity for his pets (proximal), praising their "magic"(magiques) bodies and their mystic gaze (prunelles mystiques = distal), which appears to him to drift between worlds.

Returning to Les Chats again as a whole, we can now see a repetition of themes relating to the continuum of proximal (ordered) vs. Distal (disordered):

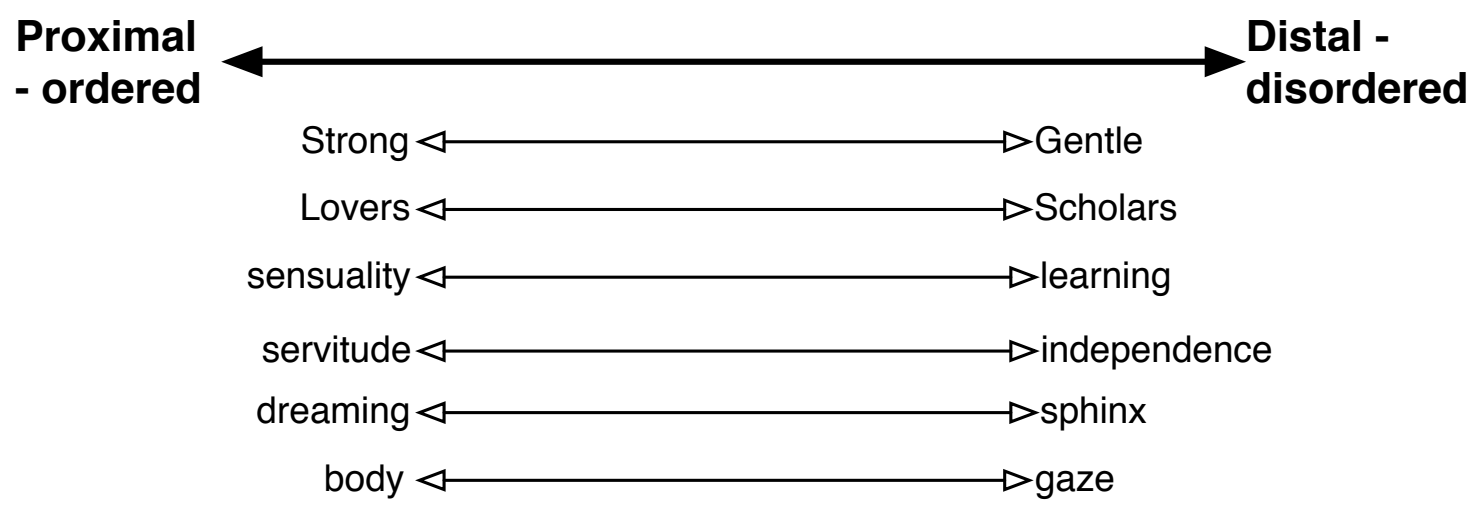

Figure 4 - Diagram of themes parallel to the semantic networks Proximal and Distal in Baudelaire's Les Chats.

Now that we can see (from the above diagram) how the themes Baudelaire references relate to the semantic networks proximal and distal, we must remember that all of these themes are supposed to coexist in the same feline. This assertion leads us to rule out either extreme (proximal or distal) as a sufficient defining characterization of cats and rather look for some concoction of the two. Resulting in an aesthetic state lacking true equilibrium, Baudelaire's observation of the distinguishing quality of cats, elevate the celebrated pet to the status of life personified (characterized by the opposition between order and disorder). 
Returning to Baudelaire's notion of beauty as always containing "a touch of strangeness", we see how our feline friends, as described by Baudelaire, follow suit; embodying both the proximal, ordered, and familiar as well as the distal, disordered, and strange. We can now see that Baudelaire's principal of beauty as asserted is consistent with his art.

But is this opposition of semantic networks exclusive to the work of Charles Baudelaire? In the following chapters I will attempt to illustrate that opposition is a fundamental constituent of the aesthetic experience and can be found in any modality (e.g. visual art, music, literature) that serves to structure the signification of its network.

\section{Narrative Dynamics in Balzac}

Le Chef-d'œuvre inconnu or "The Unknown Masterpiece" (first published in 1881) is a short story by Honoré de Balzac - a French novelist and playwright best known for his sequence of novels and plays entitled La Comédie humaine (which eventually included Le Chef-d'œuvre inconnu).

In Le Chef-d'œuvre inconnu, Balzac tells the story of a master painter (Frenhofer) who becomes acquainted with a young protégé (Poussin) while visiting the studio of then-court painter "Porbus" who is working on a painting of Mary of Egypt. During the visit to Porbus' studio, Frenhofer expounds on how the painting being worked on could be "brought to life" through a technique the "he alone" has perfected. 
Demonstrating this in front of young Poussin as well as Porbus, Frenhofer commits a few strokes of his brush to Porbus' canvas thus impressing the two artists with his skill and earning their immense respect and admiration. After this display of mastery, Poussin and Porbus eagerly beg to see the master's work, only to be denied a glimpse. On pressing the issue, Frenhofer confesses to the two artists that while he has perfected his technique, he has been unable to finish his masterpiece (the portrait of Catherine Lescault), on which he has worked for the last ten years, for lack of a suitable model. Realizing that Frenhofer will only reveal his masterpiece once it has been completed, the young protégé offers up his own lover, an unspoiled beauty (Gilette) of incomparable physique to Frenhofer as a model in order to buy himself entry to the master's most protected work. Once the master lays eyes on young Gilette, he is taken with her beauty and quickly finishes his painting in a fury of inspiration. As per the deal, Porbus and Poussin come to Frenhofer's studio to see his great masterpiece, only to be presented with a work resembling a mess of lines floating in a swirl of colors. So disappointed were the two artists that their reactions to Frenhofer's work drives the master to despair and madness resulting in his setting the painting on fire and committing suicide.

Within Balzac's story there are a few common themes: art as self-reflection, art as a rhetorical influence on perception (how art changes the way an artist is perceived), and structural parallelism. In each of these themes, as well as the structure of the work itself, our theory of entropy (as a force-dynamic characteristic of the underpinnings of cognitive aesthetics) is present. 
First, consider the characters at work in Le Chef-d'œuvre inconnu: Frenhofer, Porbus, Poussin, Gilette and the "unknown masterpiece". For Porbus, this force dynamic is defined by his aspiration as a painter towards nature (towards an ordered state) force dynamically constrained (entropic) by his limitation of skill that results in a flat "lifeless portrait".

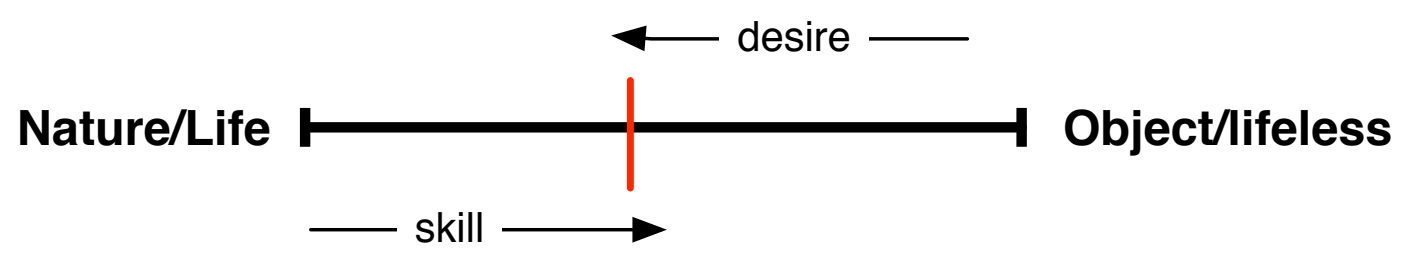

Figure 5 - The underlying force-dynamic theme represented by the character "Porbus" throughout the text.

For Poussin, it is his aspiration to become a great painter (focus on skill) that creates a drive contrary to his dedication as a lover (focus on sentiment).

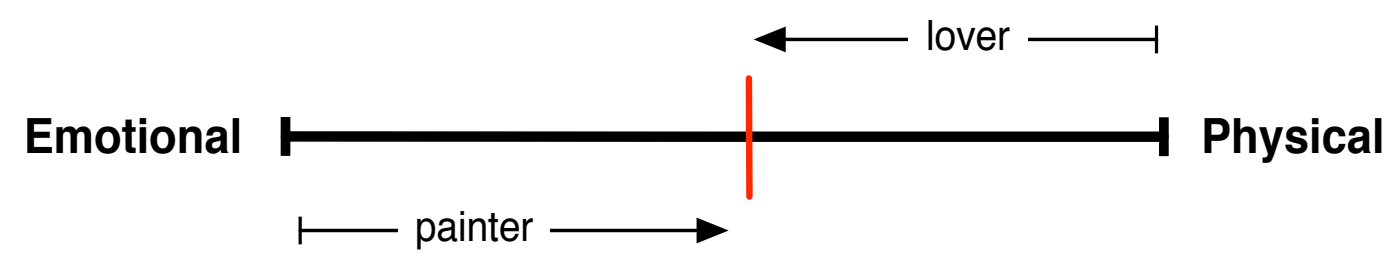

Figure 6 - The underlying force-dynamic theme represented by the character "Poussin" throughout the text.

This dynamic is substantiated by Frenhofer's endorsement for Poussin as having promise as a painter and Poussin's sacrifice of his lover for the purpose of realizing this approval.

In the case of Poussin's lover Gilette, her dynamic is created by her personification of life and purity (her natural drive towards her own wholesome state) conflicting 
with her submission to be objectified; again we see the theme of animate vs. inanimate.

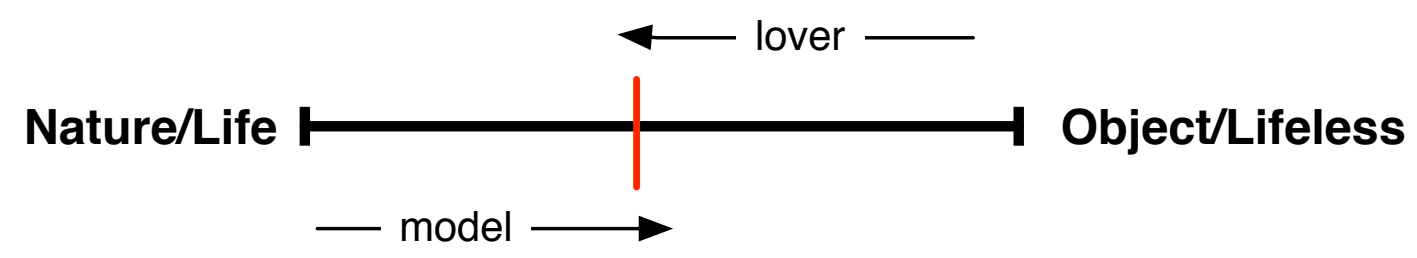

Figure 7 - The underlying force-dynamic theme represented by the character "Gilette" throughout the text.

Next, the "Unknown Masterpiece" itself as a central character can also be represented by this entropic dynamic. As it is presented, for the vast majority of the story, the "Unknown Masterpiece" exists only as a concept or hypothesis referred to by Frenhofer through his impassioned rants. This vivid characterization put forth by Frenhofer illustrates the origination of his work's "life". In this way, the point of conflict present in the painting's dynamic is defined by the painting's shift from concept (Frenhofer's lectures) to form (painting as revealed); once more, the theme of object/inanimate vs. life/animate.

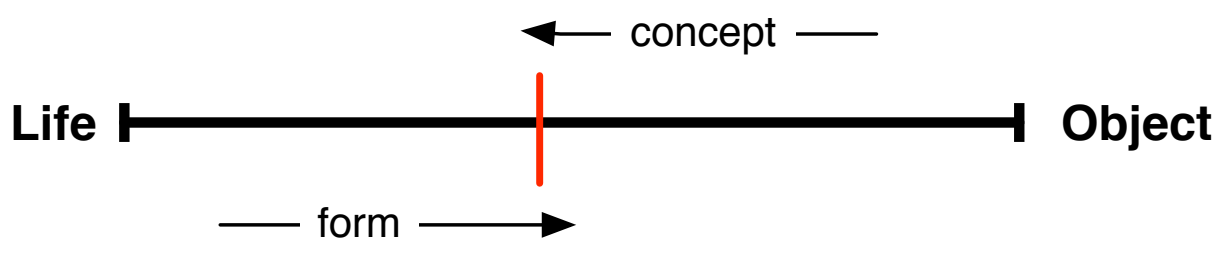

Figure 8 - The underlying force-dynamic theme represented by the character "Frenhofer's Masterpiece" throughout the text.

Last, and perhaps most importantly, we come to the dynamic of Frenhofer. In Le Chef-d'œuvre inconnu, Frenhofer is perhaps the most complicated character, 
appearing to the reader in the first half of the novelette to be a genius and in the latter a madman. In continuing with this pattern, a single element (the Unknown Masterpiece) is represented in two opposing ways; one supported by the reader's expectations based on Frenhofer's ravings (the paintings continued refinement as neg-entropic) and the other buttressed by the later revealed actuality of the paintings existence as an increasingly disordered (entropic) figure. Both of these drives are paralleled by peripheral themes corresponding to the same continuum. In the direction of order, Frenhofer's choice to paint a portrait of Catherine Lescault ${ }^{15}$ serves as a means to limit the seemingly infinite possibilities for subject matter and later the addition of Gilette, as a model figure, additionally supports this drive to limit/constrain/ and thus order the painting. Represented by a drive towards disorder are the themes of Frenhofer's decreasing sanity and reputation.

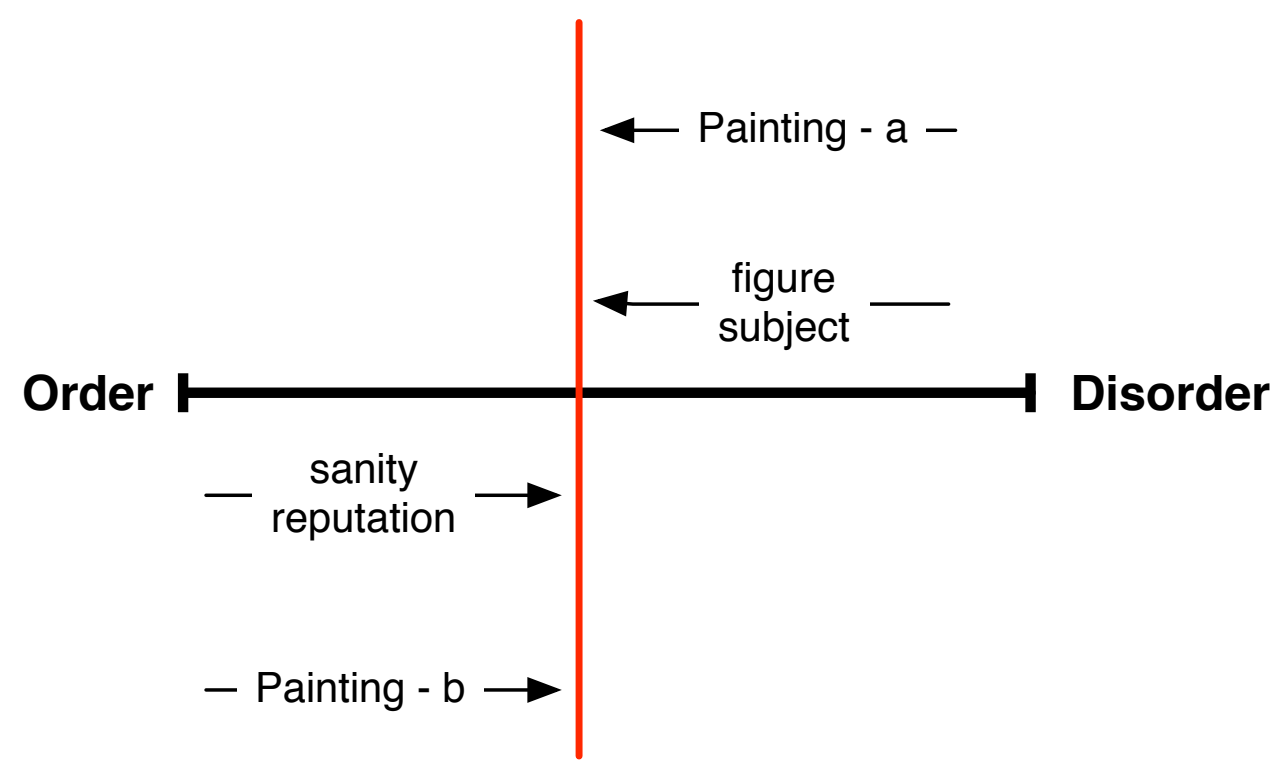

Figure 9 - in this mapping, the "masterpiece" is referred to in two separate parts "a" and "b" corresponding to the states of the painting described in the text. In the beginning, the "masterpiece" is being brought closer to "perfection", ("a") during this time; Frenhofer is seen as a genius creating a

\footnotetext{
15 Due to Catherine Lescault being deceased during the time period of the story as written, Frenhofer
} is driven to accept his need of Gilette as a substitute in order to finish his "Masterpiece" 
parallel between the painting's drive towards order and Frenhofer's positive reflection of character. The latter half of the text ("b") describes the painting as becoming increasingly disordered and again sets up a parallel, this time between the paintings drive towards a disorderly state and Frenhofer's negative reflection of character.

\section{Mapped another way:}

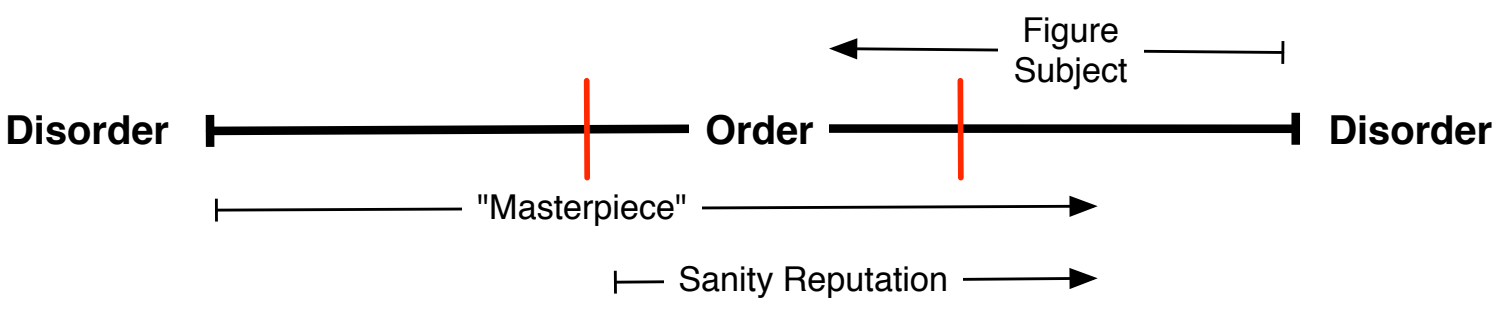

Figure 10 - This mapping had the same meaning as the one preceding, just constructed another way. The main difference between these two methods of mapping is in how the continuum is illustrated. In the preceding mapping, the continuum is shown as a gradient between two end-points, in this one, it is inferred that the continuum goes on forever, oscillating between ordered and disordered states of being.

All of the before mentioned dynamic patterns can be characterized as either a drive towards or away from disorder. This can be shown as such:

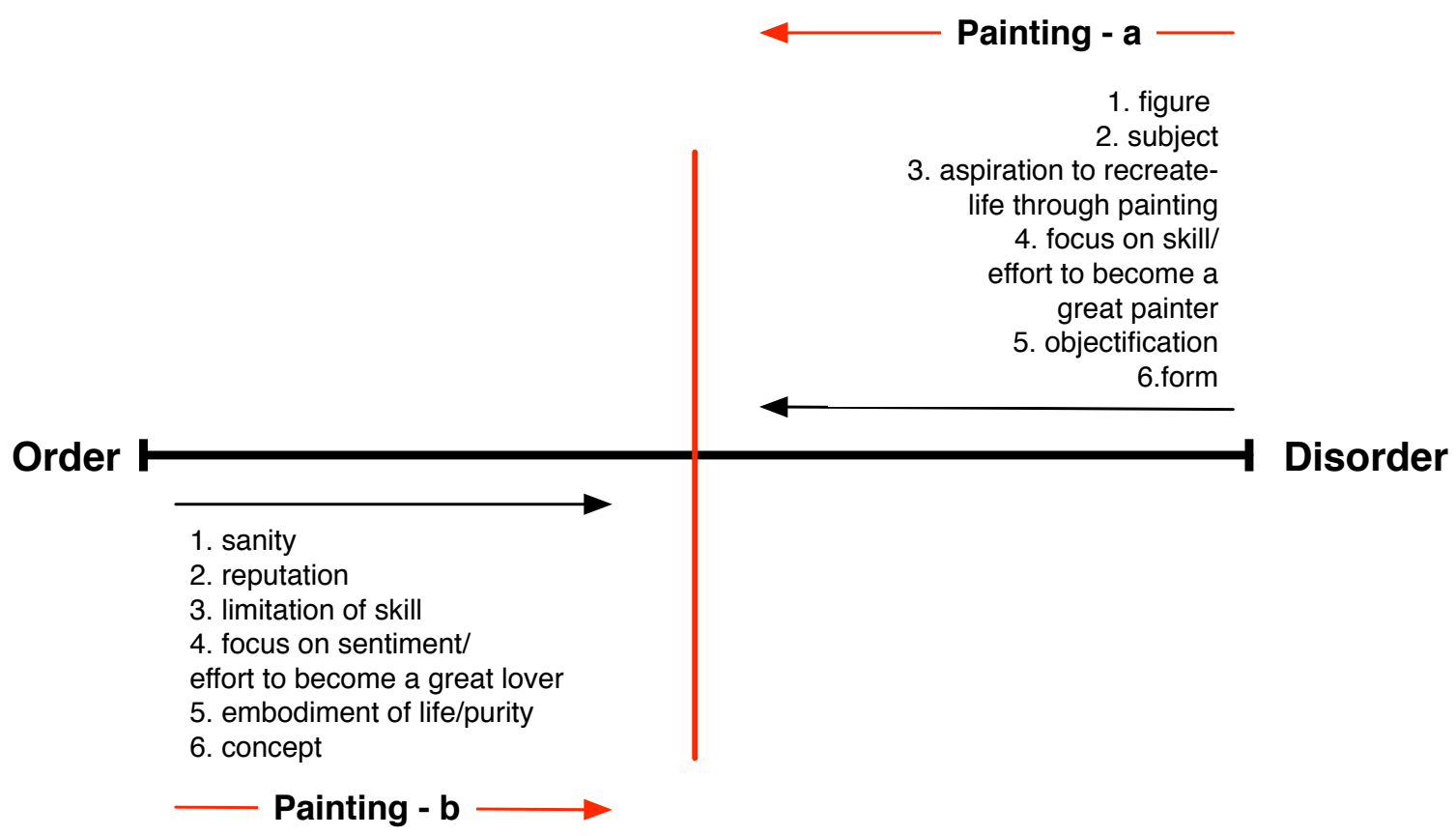

Figure 11 - Favoring, for the moment, the mapping style described in figure 7, this mapping illustrates the combined force dynamic oppositions present in the text. 
Additionally, as is suggested by the repetition of force dynamic tendencies, the structure of the narrative can also collapse into a congruent pattern. The central focus of the narrative can be reduced to the painting's drive/progression towards an entropic state (disorder) opposing the objectification of Gilette representing a negentropic drive towards a fixed state (order). The tension created by these two clashing drives indicate three points of conflict that function in parallel and represent turning points for each respective theme. The first point of conflict corresponds to Gilette's shift from subject to object; the point in the narrative where Gilette submits to being a model for Frenhofer, the second point of conflict is the unacknowledged instant in which Frenhofer's painting crosses over from driving towards order (from blank canvas to recognizable figure/subject) to driving towards disorder (continued embellishment disintegrating the form).

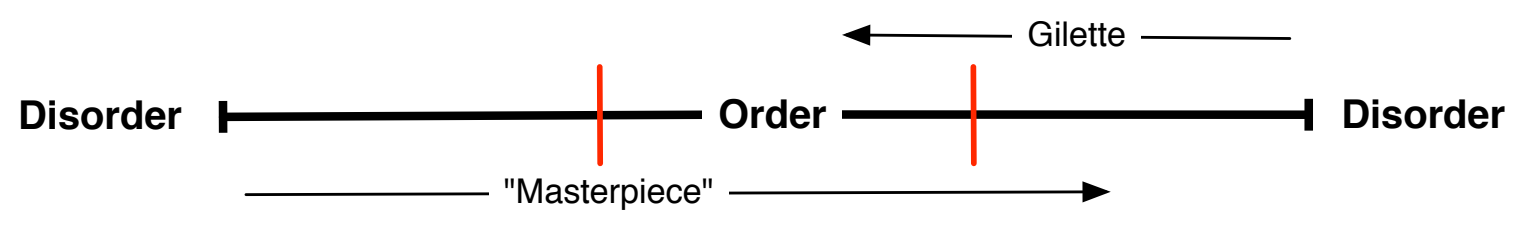

Figure 12 - In the style of figure 8, this mapping illustrates the overarching theme of the narrative structure.

The last and most dramatic point of conflict takes place in the moment where the "masterpiece" is revealed thus shifting it from concept to form.

The dynamic parallel between Frenhofer and his "masterpiece" binds the two characters causally, supporting the before-mentioned "common theme" of self- 
reflexivity (self reflection) in art. In other words, as our view of Frenhofer shifts, so too does our view of his painting. In turn, our feelings towards his painting then inform our understanding of its maker. This parallelism creates a compelling narrative where the reader can continually foreground or background the frame by which we come to understand the genius (or lack thereof) of Frenhofer's character.

The significance of self-reflexivity in Le Chef-d'œuvre inconnu is not to be overlooked as it serves to highlight the symbiotic relationship of artist and art. The notion that an artist's intentionality is in fact an integral part of the semiotic significance of any intentional work has been the source of much theorizing throughout the history of formal aesthetics. While it is not the focus of the present study to review the literature through which this issue has been investigated in more depth, it is pertinent to note that artists and theoreticians of the Post-Modern era commonly comment on self-reflexivity. In the case of the present inquiry, this notion is tractable in that the academic pursuit of a general principle by which art and nature can be reconciled has historically been one that serves to remove the intentionality of the artist from the analysis as a redundant instance of nature. This however would serve to communicate that cognition, emotion, creativity, and so on are not special or important - since nature is just uniformly causal and mentally blind - and so goes against my present assertion, which places cognition at the center of analysis. 


\section{Framing the Ontology: Reflexivity and Cognitive Semiotic Networks}

Reflexivity is further exercised when we consider the larger network that frames

our experiences and the way we come to interpret them. Per Aage Brandt uses an "ontological graph" to describe the cyclical processes at work throughout our "world"16:

4. Culture
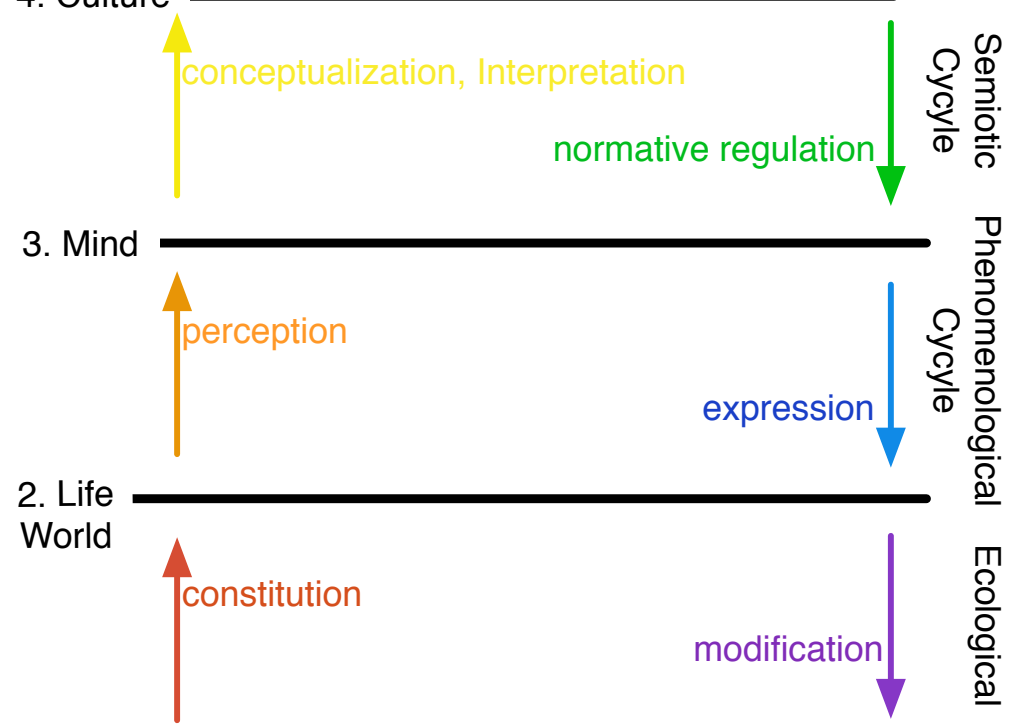

1. Physical

World

(non-accessible without

modeling or instruments)

Figure 13 - this is a recreation of the ontological graph that appears in Dr. Per Aage Brandt's working paper titled "The world. A view from within".

Beginning with the first stratum, the "physical (unseen) world" is comprised of atoms, molecules, cells, matter and so on (e.g. constitution) and can be modified by the second strata which is composed of our perceivable (from human scale) world (e.g. modification through, for example, pollution). While this "world" exists, our knowledge and experience of it is mediated by our need for instruments to observe

\footnotetext{
16 From Per Aage Brandt's working paper entitled, “The world. A view from within” from February $4^{\text {th }}, 2010$.
} 
it, and models to examine, analyze, and communicate it. The process, by which we send and receive information to and from this first stratum, is contained in the "Ecological cycle".

The second stratum, called the "life world", refers to our understanding of the world as it relates to "human scale" and contains our:

"physiology and neuro-physiology, which generate our brain and, on a macroscopic level, our mind, with its intricate interplays of memory and consciousness"(Brandt, 2010) ${ }^{17}$.

The process by which we engage socially can be described in part as the "Phenomenological cycle" which is the interaction between the perceivable world and the mind. This cycle is where experience informs knowledge and knowledge frames our interpretation of experience at the singular (personal) level.

The third stratum, labeled "mind", includes thinking, feeling, remembering and planning. As Brandt states in his working paper:

"Our mind perceives states and events happening in the pheno-world. It also consciously makes us act in such a way that we produce things in this 'outer' world, including expressions of our thinking and feeling."

It is here that we begin to get language, art, music, literature, and poetry and because of the existence of the fourth stratum (culture), these expressions can become part of a collective exchange that informs our shared "knowledge" otherwise understood as culture. Brandt concurs:

"When human minds interact and collectively create special surroundings that improve and stabilize their conditions of life, knowledge, and procreation, they transcend the level of individual minds and reach the level of what we call culture, or civilization."

17 From the working paper "The world. A view from within" by Per Aage Brandt. 
The process by which information is exchanged between the individual and the larger society as a whole, is labeled the "semiotic cycle" and is characterized by the use of sign systems and language as the most "prominent manifestation of culture"18.

How this network comes to inform our understanding of reflexivity is that, as we can see in figure 13, the mind communicates it's perception of the world through the use of sign systems (e.g. art) which then becomes part of the accumulative understanding of many minds (e.g. culture) which, in turn, adds to ideas we "receive" from the semiotic cycle that inform and or stabilize our initial view of the world.

Let us now explore an example from E.H. Gombrich that will serve to illustrate how reflexivity can inform our assumptions about the value of an artist's intentionality in a work of art.

\section{An Actantial Model of Monet's Roven Cathedral}

In Part One of E.H. Gombrich's, (an Austrian-British art historian) pinnacle book; Art and Illusion, the discussion of Monet's Rouen Cathedral, leads Gombrich to reflect on why the painter expressed the effect of light hitting the façade of the cathedral;

18 Brandt, 2010, working paper. 


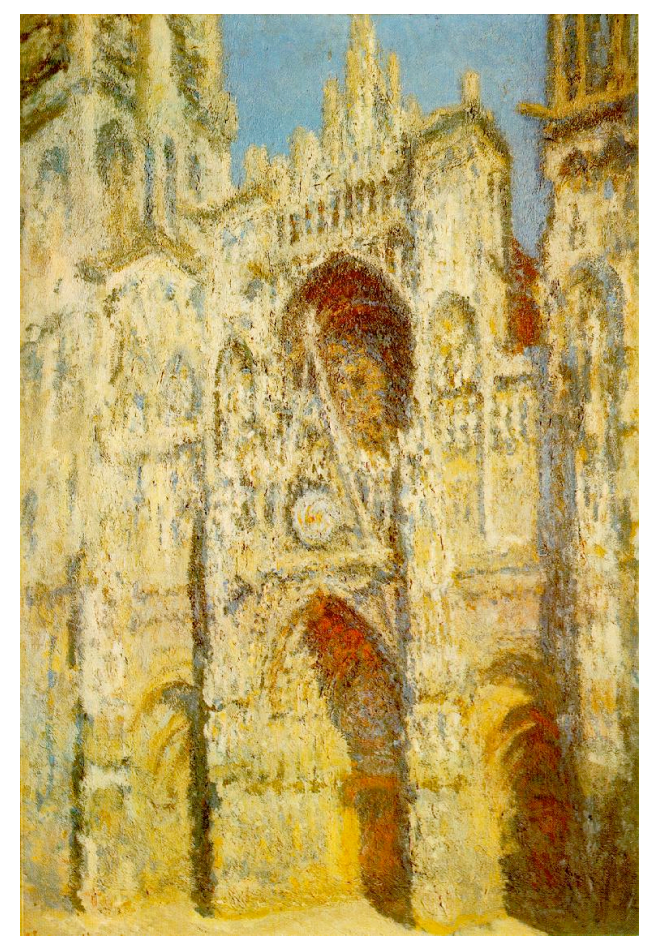

Figure 14 - Claude Monet's Rouen Cathedral

"The experiments of the impressionist painters have convinced us that these limitations of the medium can be overcome: a painter like Monet can suggest the effect of the midday sun by exploiting the dazzle that results from its glare..."(Gombrich, 44)

In this statement, Gombrich makes the assertion that Monet's approach to painting stands as a singular example of an artist's ability to overcome the limitations of his chosen medium through shifting the focus of their artistic intent to that which is better captured through their particular skills and/or instruments. Considering this claim, we are lead to understand Gombrich as imagining that Monet's original desire had been to capture the unaffected physical appearance of the cathedral, and that his choice to instead capture the effects of light on its façade represents this "shift of intent". 
Taking a cue from well-known Structuralist Algirdas J. Greimas (1917-1992), who argues that the perception of oppositions underscore the "elementary structure of signification"19, we can employ an actantial narrative model to illustrate Gombrich's interpretation of Monet's approach to art. But, first, let us explore how the model functions:

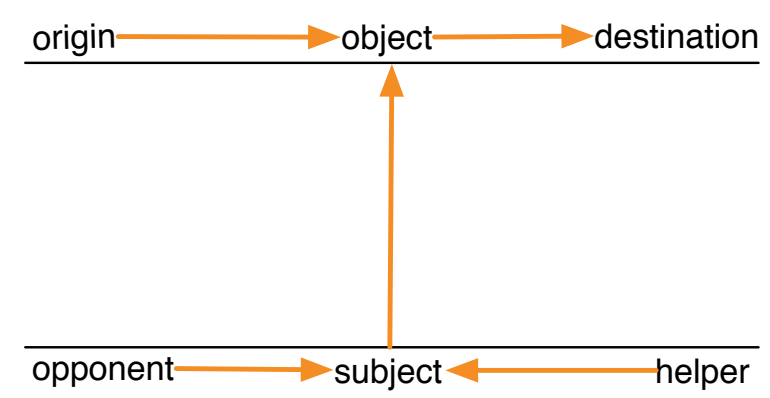

Figure 15 - generic actantial narrative model describing the placement of variables.

In this model, there are three relationships described: the subject/object, origin/destination, and the opponent/helper. First, the subject goes in search of an object; this object can be a material item or an abstract concept. Next, the subject is either aided by the helper, or obstructed by the opponent on their way to the object. Last, the origin is an "actant" that functions as a catalyst for change, with the destination denoting the "change state". 20

Let us now apply this model to our discussion of Gombrich:

${ }^{19}$ Structuralism and Semiotics, 2003 and also Structural Semantics, 1966

20 Dictionary of Semiotics, 2000 


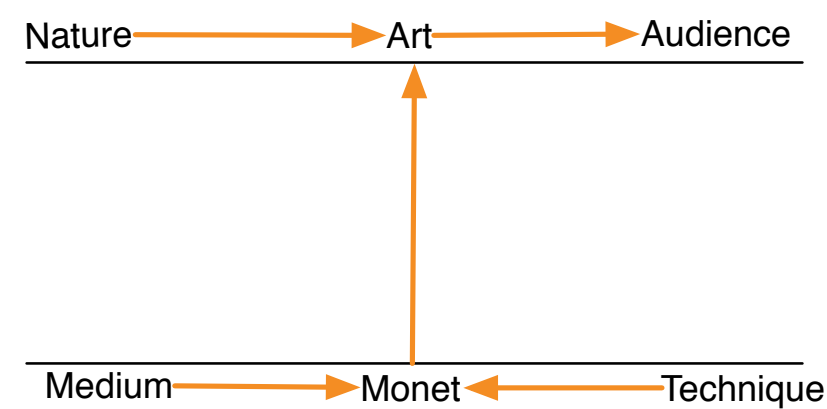

Figure 16 - Actantial Narrative Model of Gombrich's view of Monet's approach to art.

From this illustration, we can see that Monet, as the artist, is placed in the subject position, with his object being "art". We also see that, as so prompted by the quote above, Monet's helper is his technique and skill as a painter, while his opponent is the medium of paint and canvas, which limits Monet's artistic expression. Last, our reading of Gombrich leads us to place (the mechanism of) "nature" as the origin, and the "audiences" as the destination. This interpreted placement helps us to visualize the relationships at work behind Gombrich's theory of art. For Gombrich, art is the transposition of nature to an audience by an artist through the use of technique and in spite of the chosen medium.

To this, Gombrich agrees:

"What a painter inquires into is not the nature of the physical world but the nature of our reactions to it. He is not concerned with causes but with the mechanisms of certain effects." (Gombrich, 44)

Referring back to our discussion in section $\mathrm{XI}$, the inquiry of a painter as described by Gombrich in this quotation would reside in the phenomenological cycle taking place between the perceived world and a single mind. This may indicate that Gombrich thinks that nature (as perceived) is beauty, and so sees no need to further explore the reasoning behind intentionalities that support anything 
other than the decision making that leads to the most "successful" representation of the natural world possible by a particular artist.

Judging from the quotation above, Gombrich believes that what Monet captured in his Rouen Cathedral, was his attention to the mechanism of light as a solution suggested by the perceived resistance or limitation of his medium (oil paint, brushes).

I posit a different interpretation:

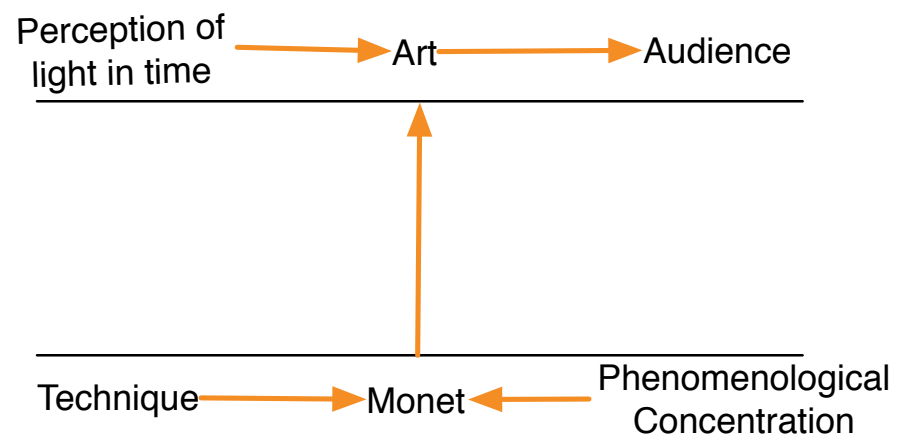

Figure 17 - Actantial Narrative Model of Monet's approach to art

What is different in the above model is the helper/opponent relationship, and the role of the "origin" variable. Instead of nature being the contained objective for art's delivery to an audience, I instead submit that the motivating idea behind Monet's Rouen Cathedral is Monet's perception of light in time. Whereas Gombrich focuses on technique, Monet places his focus on the phenomenological. This idea is supported by the existence of the "Rouen Cathedral series" which depicts the same subject at many different times (e.g lighting conditions) of the day. Also, instead of casting "technique" as the helper (as Gombrich does), it is placed here as the opponent: something to overcome. Considering for a moment, the early work of 
Claude Monet that depicts scenes in a highly realistic sense a la the École de Barbizon (e.g. French landscape and outdoor art) movement, it can be assumed that if "nature" is what was being strived for, than Monet's artistic skill could have easily achieved it through mastery. However, I assert that this was never the objective; that instead, the "helper" in our model, is better served by Monet's own Phenomenological concentration which allowed him to focus on his perception of light (cognitive) in spite of its material relationship to nature (temporal), thus helping him to transcend the constraints of his formal training.

This shift in semiotic perspective can be explained further: Consider first, how we comprehend the experience of looking at a painting in a museum: as we gaze at the image hanging on the wall, our understanding of it is framed by our somatosensory experience of our body in a particular space, a situational understanding that we are looking at a piece of art in a museum, a cultural understanding that informs us that the work of art we are looking at has some value, importance, or relation to art history, and a cognitive understanding that allows us to process this information as a whole schematic gestalt.

With the whole of this gestalt framing our experience, we can attend to the work of art before us in two ways: one where we experience the work as an image and as an object separately, and the other where we experience it as a blend. While most viewers of art (who do not possess formal training) immediately respond to the cohesive work as a whole (the blend), those who have undergone aesthetic indoctrination might choose to attend only to the construction of an art object (the 
direction of the brushstrokes or any particular techniques for example), while ignoring the content (the subject or image depicted) or vice versa.

In order to more clearly illustrate, consider the following mapping:

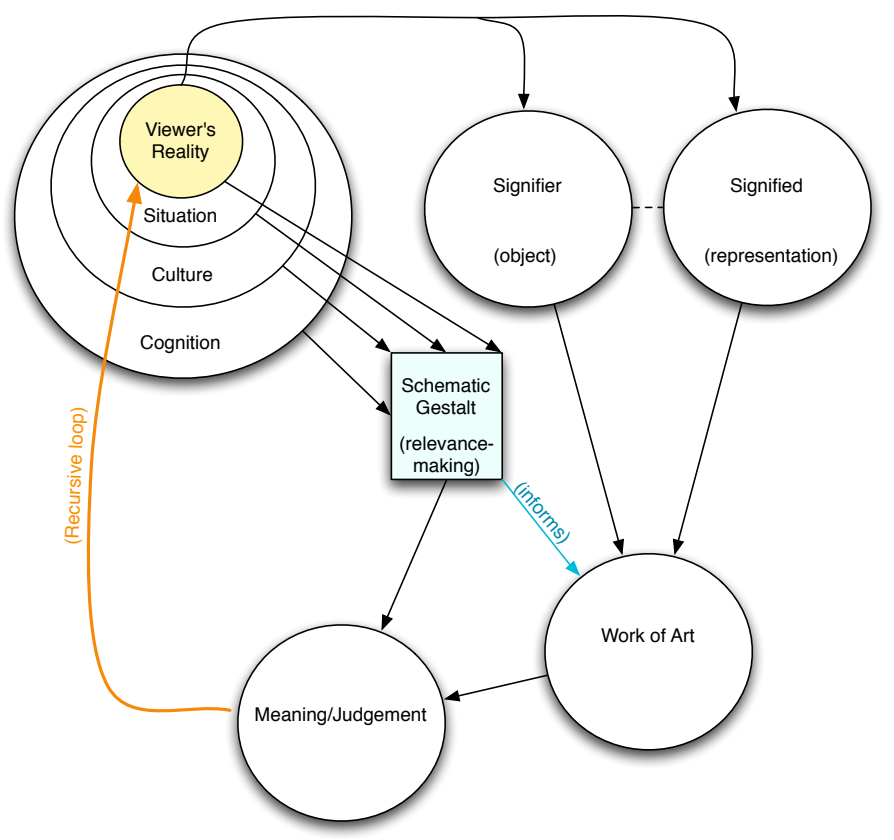

Figure 18 - Semiotic blending network illustrating the duality of art as both signifier and signified with our reactions to it as a contributor to culture.

Turning now to Monet, we can assume that his personal history as a trained artist would allow for him to be able to oscillate the attention of his aesthetic experience between the blend and the input spaces (signifier and the signified independently). This ability would have allowed Monet to have, first, seen Rouen Cathedral at once as a stone architectural structure in space, and a surface interacting with present lighting conditions, and, second, as a fleeting scenario (commenting on the impermanence of the lighting condition) that when seen through the gestalt of 
framing schemas ${ }^{21}$ (for example - illumination being a cultural symbol of the divine) creates an ever more relevant, inspired, and meaningful subject ${ }^{22}$.

Looking at the present study in this way, we can see how Monet's choice to depict Rouen Cathedral as a wall of reflected light could have represented an attempt to create a meaningful work of art in addition to creating a beautiful representation of his experience, and not just a way to "overcome" perceived shortcomings. We can also further dispute Gombrich by referring back to his earlier quote where Gombrich asserts that artists only attend to the "nature of [their] reactions [to the physical world]". This would mean that only the "meaning/judgment" space was being utilized for artistic content. This is of course absurd, as it would serve to discount any and all art whose function is to comment on the nature of construction, cultural irony, or the avant garde. Take for example Richard Serra's "Splash" pieces made between 1968 and 1970; in these works of art, Serra highlights the "Signifier" space by drawing the attention of the viewer to his medium, which in this case is [molten] lead. From the viewpoint of the artist as maker, Serra is not observing naturally occurring phenomena, but is rather using his knowledge of the material (from the schematic gestalt) to speculate about how his actions (process) will affect the material outcome. By doing this, Serra is taking information from his frame of experience (Viewer's reality), combining it with the "Signifier" space, which favors

\footnotetext{
21 The "relevance-making schematic gestalt" in the case of Monet, is most strongly asserted as the artist's understanding and experience of the phenomenological.

${ }^{22}$ In Chapter 8 of Gombrich's book, Art and Illusion, entitled "Ambiguities of the Third Dimension", Gombrich states "Any picture, by its very nature, remains an appeal to the visual imagination; it must be supplemented in order to be understood". While he applies this statement to his discussion of "perspective", if we extended his intension to also apply to that which is referred to in the present text as the "schematic gestalt", his observations could have supported the foundations of cognitive semiotics.
} 
material over content, and exporting it directly to the "Meaning/Judgment" space.

The artwork in turn funnels back into the nested frames of "reality" (schematic gestalt) as it becomes a part of the culture that contributed to the conditions that produced Serra's art in the first place.

While the importance and salience of Gombrich's work is not in dispute as a whole, His generalization of Monet's motive for creating his art lead to incorrect notions of the cognitive aesthetic experience.

\section{Greimas and the Semiotic Square}

The actant model introduced in the previous section helped us to discern the difference between the perspectives offered by Gombrich and that which is asserted in the present study. But, the model lacks the dynamics representative of the open system we have come to characterize art (as it occurs in culture) by. This, by no means, undermines our previous example, but rather calls for an extension of it.

In the introduction of Algirdas J. Greimas' seminal work entitled, Structural Semantics (1966), Ronald Schleifer (George Lynn Cross Research Professor of English at the University of Oklahoma) writes:

"The semiotic square is the logical mapping out of structural possibilities: for any content which can be understood as itself analyzable into binary oppositions ( $S$ vs. non $S)$ the square repeated and super-posed, will exhaust the logical structural relations between its minimal elements (Greimas, xxxiii)."

The semiotic square, then, is a model illustrative of the dynamics inherent in any semantic structure, which is framed by a schematic gestalt that contributes to its 
network the relevance of its context. I posit that this model, as a logical (and dynamic) extension of Greimas' original actant model serves well as our final methodological approach to the identification and analysis of the cognitive aesthetic experience.

Let us then, examine the elements at work within a semiotic square:

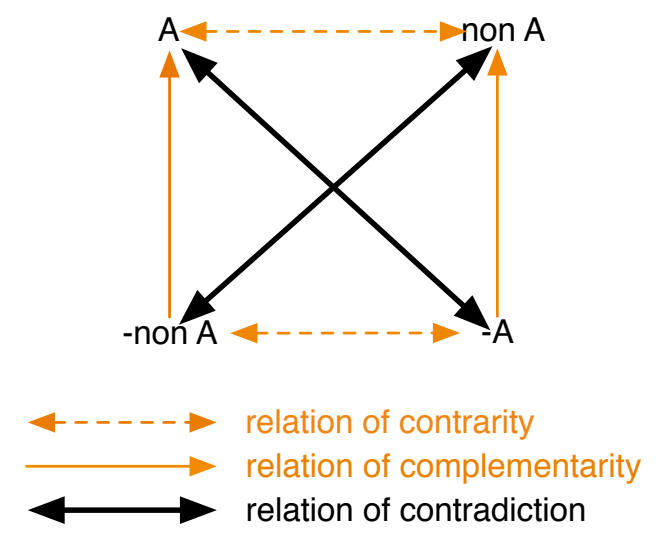

Figure 19 - Logical Negation in Greimas' semiotic square

As is seen in figure 19 above, the simple elements involved in a semiotic square can be described as two types of binary relations. Greimas defines these relations in his book Semiotics and Language: An Analytical Dictionary (1979), as such:

"The first, of the type A/-A, characterized by the resultant opposition of the presence and absence of a definite trait, and the second, or the type non $A /$ non $A$, which manifests to some extent the same trait, present twice in different forms. (Greimas, 308)"

This model is static and serves to describe the "deep structure" of a situation or narrative in abstract terms. In order to model the dynamic relations involved in any narrative structure we must think back to the elements involved in our Actant Model from Section 11 where we have the "helper" and the "opponent". Adding them to our semiotic square format in place of one of the set of binaries, we can 
begin to see how the opposition in the model function to circulate the subject or object placed within this network:

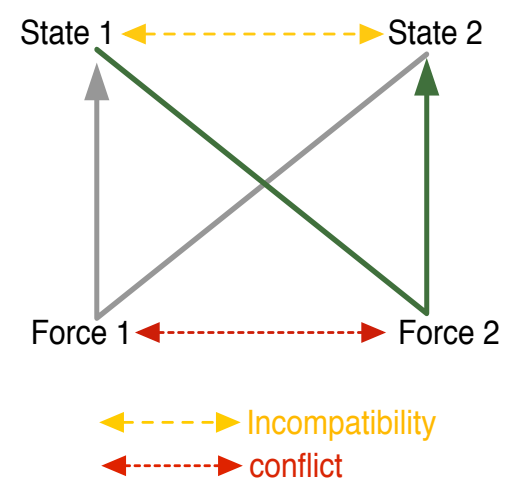

Figure 20 - Dynamic version of the semiotic square model described by Greimas- with key

Like the Actant Model described in Section 11, the Dynamic Semiotic Square illustrates narrative structure. Wherein Figure 19 illustrates the logical opposition of the elements present in the narrative structure, the model in Figure 20 shows how those oppositions function dynamically between connected elements (e.g.narrative). In the above illustration (Figure 20), State 1 and State 2 could be considered placeholders for the elements from our actant model; origin, and destination, but could also be filled with other incompatible components of a narrative as needed. Additionally, Force 1 and Force 2 could be construed as the helper and opponent elements (also from our actant model), but could be filled as well, with other components that represent a central conflict between the connected elements being analyzed. The way these States and Forces relate then, is that the helper of State 1 is the opponent of State 2 and vice versa ${ }^{23}$.

${ }^{23}$ Likewise, the opponent of State 1 is the helper of State 2. 
With this model in mind, let us explore another example of dynamism in art.

\section{Embodied Dualities: Wallace Stevens}

"The imperfect is our paradise."

$\sim$ Wallace Stevens, 1942

In 1942, prominent American Poet Wallace Stevens (1879-1955) published his fifth collection of poems entitled Parts of a World. While not his most critically acclaimed volume, Parts of a World shows a matured and refined Wallace looking to reconcile the horrors of the Second World War with the continuation and forward development of cultural and social values. While it is pertinent to note that the force-dynamic themes of order and disorder are sustained throughout the poem (and also present and foregrounded in the context of the poem's writing), the focus of the present section will be on the semantics of the text independent of its relationship to its historical context.

In examining a single representative poem from the collection: "The Poems of Our Climate," I aim to illustrate how the force-dynamic themes of disorder and order (or entropy and neg-entropy) are again (as they are by other artists in many modalities) consistently represented (both intentionally and subconsciously) as the lodestar of art: a paragon form.

\section{The Poems Of Our Climate}

I.

1. Clear water in a brilliant bowl,

2. Pink and white carnations. The light

3. In the room more like a snowy air, 
4. Reflecting snow. A newly-fallen snow

5. At the end of winter when afternoons return.

6. Pink and white carnations - one desires

7. So much more than that. The day itself

8. Is simplified: a bowl of white,

9. Cold, a cold porcelain, low and round,

10. With nothing more than the carnations there.

Beginning with the first stanza (above), Stevens offers us a vessel: a "brilliant bowl"24 that contains "clear water" and "pink and white carnations". Right away we are prompted to consider dualities: the container and the contained, the pink and the white, the brilliant and the dark (implied), the clear and the particulate ("snowy"). Without heavily researching the semantics of these dualities, we might interpret the vessel (bowl) as a reference to the (female) body ${ }^{25}$ and the "clear water as the spirit contained within ${ }^{26}$. Upon further research of the symbolic meaning of pink and white carnations, I came to find that the etymology of the word "carnation" was derived from the Latin word "caro"(nominative case) or "carnis" (genitive case) meaning "flesh" referring to the flower's original pink color. This was later translated (according to the OED) into English as "incarn" or "incarnacyon" (incarnation) meaning the embodiment of spirit, thus referring to the incarnation of temporal "God-made" flesh. From this meaning, we might assume ${ }^{27}$

\footnotetext{
24 Brilliance is a perceptual state implying the presence of light - i.e. spirit.

25 The notion of the body, particularly the female body, as a vessel, predates modern civilization and harkens back to primitive societies where fertility figures were often represented in the form of casks or were painted on the sides of vases and bowls symbolizing the woman's womb. This idea was later explored philosophically by Descartes who referred to the body as the vessel for the mind.

26 "Clarity" as a visually perceived phenomena (obviously related to sight, the eyes) can be seen as a reference to the mind; a proverb illustrating this might be: "the eyes are the windows to the soul" often attributed to William Shakespeare.

27 The notion of the color white as symbolizing purity, is not a new idea; its semiotic instantiation can be found in numerous cultural traditions that serve to celebrate purity and rebirth - e.g. weddings, baptisms, tribal rites ect.
} 
that the significance of the white carnation then, is a reference to the ethereal pure spirit; unembodied 28 .

The second line begins a new description - this time laden with metaphor. ${ }^{29}$ Stevens begins with a room (earthly, temporal), again pulling the theme of containment through his poem. This time, he shifts his attention to more temporal aspects of his perception, that of light (the contained, intangible). ${ }^{30}$ In describing his experience of the light in the room ("snowy", particulate), Stevens begins to make a point that he will work to clarify throughout the reminder of his poem: the necessary pursuit of light/perfection (enlightenment, spiritual awakening, transcendence) undeterred by the constraints of corporeality. The way he delivers the message of his poem is through carefully chosen, perceptually observed dichotomies (i.e. a bowl of water) and their implications (bowl=vessel, water= purity/spirituality) as multi-faceted symbolic paradigms. After establishing his theme of spirit/body duality in the first couple of lines, Stevens goes further (in lines 6-7) as to posit an evaluation of this orderly state of being; that it is not enough to fulfill desire, that the revelation of self, the simplification of the delineated divide 31 does nothing to subdue the inquisitive and unyielding nature of mind, and that this deficiency leaves one cold, and absent.

\footnotetext{
28 The symbolism of the color white is often understood as relating to purity, or cleanliness. In fact, another word that can be traced to the same Latin root - is the word "Candidate" which is significant in that in Ancient Greece, those running for office wore white as a symbol of their "purity" before taking office.

29 The light in the room is being compared to snowy air, which is compressed into a greater metaphor comparing the observable phenomena to that of the duality of man.

30 The presiding theme of the first stanza is the establishment of a spirit/body duality, the vessel and water, the room filled with light, and the pink and white carnations all function as semiotic referents to this essential metaphor.

31 Between body or form and spirit or expression - i.e. text and meaning
} 


\section{The Poems Of Our Climate}

II.

11. Say even that this complete simplicity

12. Stripped one of all one's torments, concealed

13. The evilly compounded, vital ${ }^{32}$

14. And made it fresh in a world of white, ${ }^{33}$

15. A world of clear water, brilliant-edged ${ }^{34}$

16. Still one would want more, one would need more

17. More than a world of white and snowy scents

In the second stanza, Stevens introduces the obstacle or conflict (contrary) he aims to overcome, ${ }^{35}$ which is that both mind and body strive (out of desire and necessity) at the same time, towards simplicity (cold) and complexity (hot), illustrating man's propensity towards futility (it is impossible to be in two states at once). This theme can be described by desire circulating though a system defined as the conflict between the self as duality or essentialism (e.g. body and spirit) and the self embodied or existentialism ("evilly compounded, vital I"), instantiated in either a simple (order) or complex (disorder) state of being.

\footnotetext{
32 "Evilly compounded" - turn from the divine or transcendent, and transmuted into physical form - the vital (living) "I" - an unacknowledged duality of self.

33 Return to purity, reborn

34 It is often believed that the surface/edge of water is the divide between the physical and the spiritual realms, brilliance attributed to the edges (presence of light) implies an emphasis on the connections BETWEEN the spiritual and the temporal.

${ }^{35}$ The counterfactual described is characterized as a situation in which complete knowledge is obtained, allowing the individual to order and understand the grand design of man's duality, which presumes to relieve one of the torments of ignorance.
} 


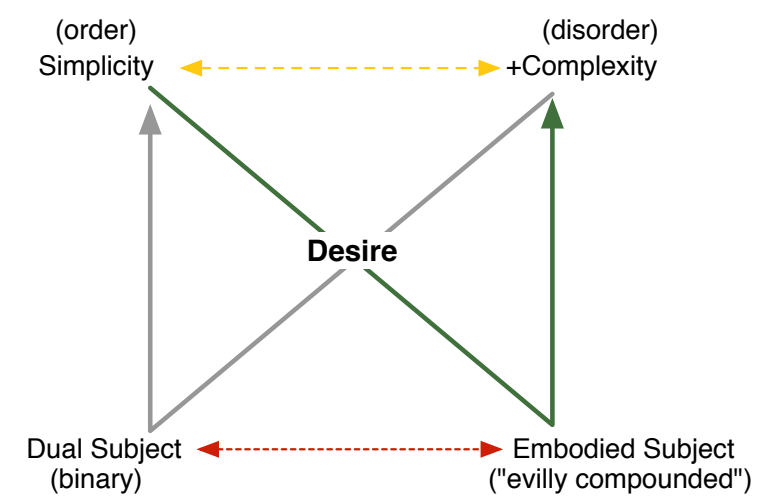

Figure 21 - Greimas' Semiotic Square depicting the dynamic structure of Steven's poem “The Poems Of Our Climate".

In this diagram, desire is placed as the dynamic element that circulates though the system defined by two states of being (identified by the yellow arrow); simplicity and complexity, and two opposing forces (identified by the red arrow); the dual subject and the embodied subject. These components function by creating tensions (along the yellow and red arrows), which serve to set the main element "desire" oscillating through the system along the grey and green lines in a never ending "figure 8".

\section{The Poems Of Our Climate}

III.

18. There would still remain the never-resting mind,

19. So that one would want to escape, come back

20. To what had been so long composed.

21. The imperfect is our paradise.

22. Note that in this bitterness, delight,

23. Since the imperfect is so hot in us,

24. Lies in flawed words and stubborn sounds.

The third and final stanza imparts a reconciliation of the mind and body, deposing the once-salient conflict with resolution. While Stevens allows that the mind, removed of the torments of the flesh (through understanding of duality), will continue to pursue new heights of transcendence, he propounds that we would 
want to return (to the corporeal, to embodiment), that the investment we have in our composed viewpoint as unique and valuable would draw us back from the extremes of metaphysical being ${ }^{36}$ to find ourselves once again at a point of conflict between a familiar opposition. ${ }^{37}$ This time it is viewed through a retrospective shift in viewpoint. This shift is exquisitely expressed in line 21; "the imperfect is our paradise", which identifies the misinterpretation of the mind/body conflict as one focused on a static goal rather than an undecided process (which is now revealed to us as primary). It is here in this line, that Steven's recognizes that the oscillating element desire identified by our recent mapping, is the paradise he has searched for; not the static notion of some divine ideal of perfection.

While Stevens' goes on to admit a sort of disappointment (line 22), he comes to revelation ("delight") in recognition of the removal of futility from the pursuit of an ideal. 38 The "imperfect" which is our paradise is also the wavering desire of man (line 23), and that descriptions or dialogues that describe "perfection" are inherently flawed as products of an imperfect creator - humankind (line 24).

The theme of duality is repeated through the medium of poetry in that, as with any mode of communication, it can be seen as an externalization of an internal process: experience and expression, text (symbols/signifier) and meaning (concept/signified), the phenomenological and the semiotic. This observation is fitting for Stevens who was known as a gifted abstractionist who deliberately

\footnotetext{
36 Detachment from the physical body, disembodiment

37 the self as a Cartesian duality and the self as an embodied one.

38 If an ideal is characterized as perfect form - the conflict represented by our embodiment constricts our ability to attain like perfection, thus distinguishing the pursuit of an ideal as futile. However if the ideal is characterized as imperfect - the objective can be seen as attainable in that it mirrors the conflict of our own duality.
} 
crafted his writing to reflect his interest in multi-faceted themes. ${ }^{39}$ In light of this observation, it is worthwhile to consider the analysis of the present poem as a whole text; an expression of the writer's phenomenological worldview, which reveals to us, the complexities of Steven's own aesthetics.

\section{Cognitive Semiotic Blending: A closer look at Wallace Stevens}

In chapter 10, I introduced an ontological graph (Figure 13) put forward by Professor Per Aage Brandt that served to illustrate how the processes at work throughout our world integrate with our cognitive understanding of it. With the semiotic blending graph below, I aim to expand on the before-mentioned ontology by integrating the phenomenological and experience-based worldview with that of the semiotic and aesthetic view.

39 Many of the themes in Stevens' poetry can be read on more than one level, sharing referents between semantic elements. 


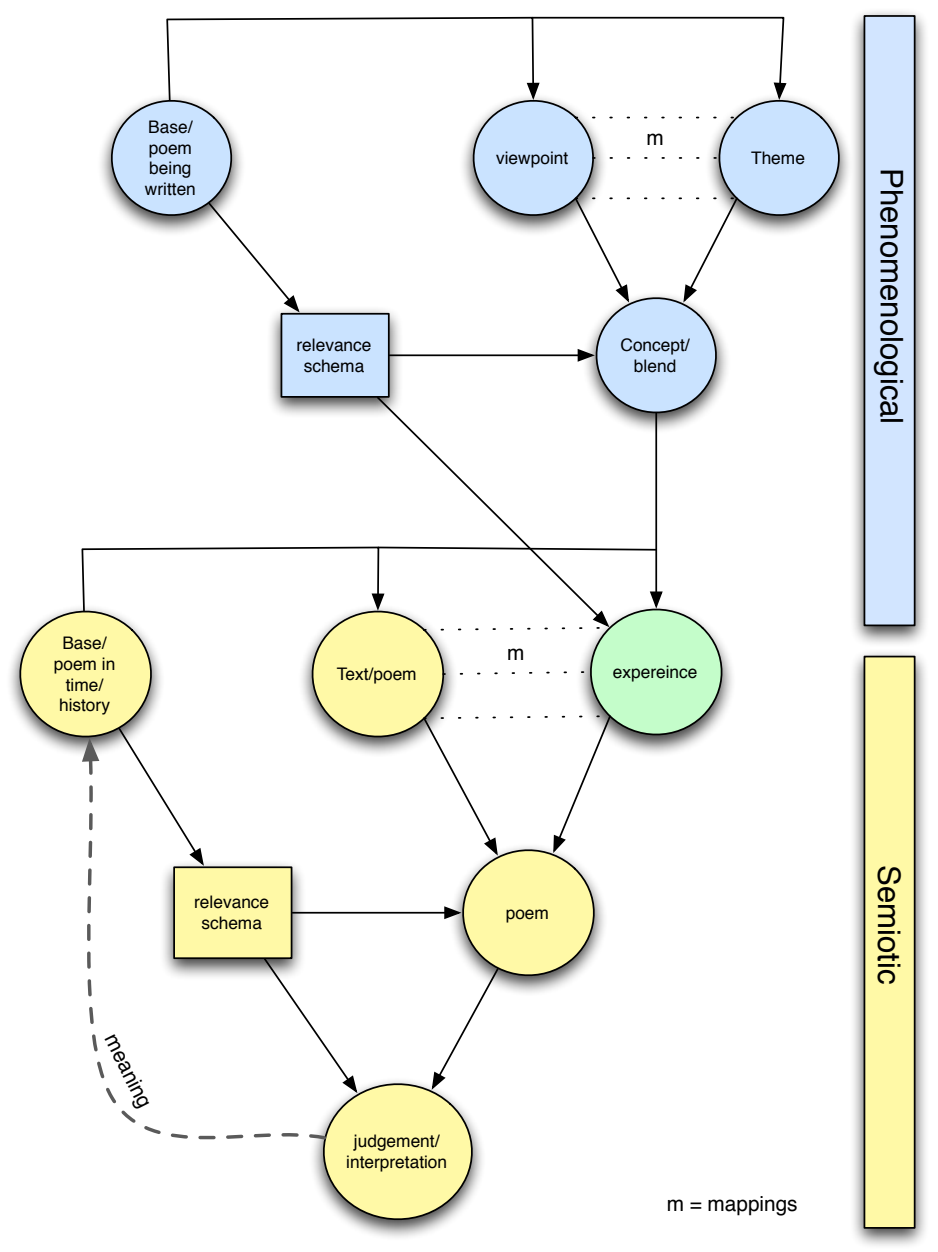

Figure 22 - Proposed semiotic blending cascade for "The Poems Of Our Climate"

The above graph represents a detailed illustration of the cognitive underpinnings of the Wallace Stevens poem before examined. Reading this diagram from the top down, we begin in the (blue) phenomenological layer with the "base space" on the top-left. In this space, is Stevens the writer endowed with all the knowledge and experience he had at the time of writing the present poem as well as his poem in the context that it was written; World War II era America, perhaps in Stevens' home, or office, and the entirety of its circumstance. This is the layer at which research takes place, where we collect the possible socio-cultural material of potential relevance to 
the poet and poem. This space acts as a background "base" or anchor for the rest of the mapping in the phenomenological layer. From the base space, two input spaces are derived, here: "viewpoint" (Steven's perspective in context/experience) and "theme," which refers to the theme/image evoked in the poem - in this case, the observed dualities. These two input spaces are then combined to create a blended space in which Stevens' applies his knowledge and experience of the perceived thematic phenomena to his present viewpoint. The "relevance-making schema" is an export of relevant experience and knowledge from the base space in gestalt format. Included in this schema is the network illustrated in chapter 12 as the semiotic square, which describes the nature of the opposing states and forces throughout the poem, and contributes to Steven's general philosophy. In order to provide stabilization, this "relevance-making schema" acts on the blend by informing the judgment or evaluation (above seen as a green "experience" space) of the structural network describing the creation of the poem.

In considering the nature of expression as originating from the phenomenological and moving toward the expressive ${ }^{40}$ (externalization of an internal cognitive process) we must continue our mapping into the semiotic (expression) layer. Taking our evaluative (green) "experience" space as an input (thus creating a cascade of blends) informed by a new base space characterized by the poem as an object (book) in time (not just at the time of writing, perhaps instead our present time of reading) and paired with a second input space filled with the text of the poem. With the inputs defined in the semiotic space, we next move on to the blend,

\footnotetext{
40 Refer to chapter 10 for more information on the process of communication between layers of the ontological graph (figure 13) referenced.
} 
above labeled (yellow) as "poem". This is the poem as a representative and meaningful text irrespective of our (as readers) experience or interpretation of it. The experiential quality of the poem comes into play again with the help of the "relevance schema" which this time, is defined by a semiotic square illustrating the nature and dynamics of Steven's aesthetic. The "relevance schema", offering stabilization again to our ("poem") space, is taken along with it, as an input for a final blend in which we come to some understanding of what we have just read.

What is informative to note, is that through the visualization offered by the preceding diagram, we can now see how the writer's experience that informs the text is separate to, but crucial for, the formation of symbolic expression and meaning generation. This divide between and articulation of the phenomenological (worldview) and the semiotic (aesthetics), the experience and the expression, the text and its significance, (the mind and the body) is an elegant expression and mirror of the poems' inspiration and message together as one, multifaceted abstraction.

As we have seen, the opposing forces of order and disorder, semantically communicated through the use of dichotomies and dynamic schemas, represents evidence of the cognitive networks that (inform and) are employed by the author or artist in order to communicate the multifaceted nature of (his) embodied experience. In addition, the structure of the poems' message paired with that of its creation and/or writing also indicates a force dynamic pattern characterized by the opposition of the embodied self and the self as dualism. This force dynamic opposition, which is understood as the ever-present abstraction of order and 
disorder, represents a strong example of the cognitive underpinnings of the aesthetic experience.

\section{Semiotic Blending and Similes: Loren Eiseley}

In an effort to illustrate, though repetition, the effectiveness of the methods presented in this text, I offer another example taken from American anthropologist and natural science writer Loren Eiseley (September 3, 1907 - July 9, 1977) who late in his life distilled his vast knowledge of science and nature into poetry. Notes of an Alchemist, the title poem from his book of the same name published in 1972 evokes many of the same themes previously discussed and serves as a fine example with which to work the methodology before outlined. Here is the text:

Notes of an Alchemist (Loren Eiseley, 1972)

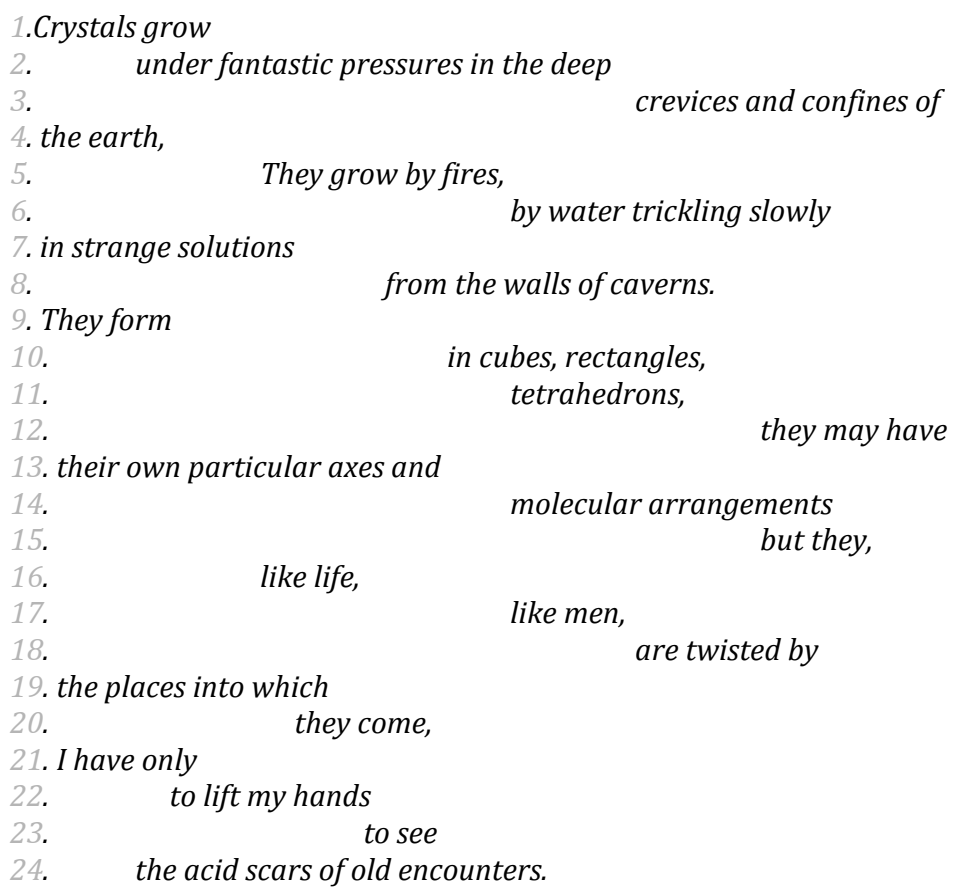




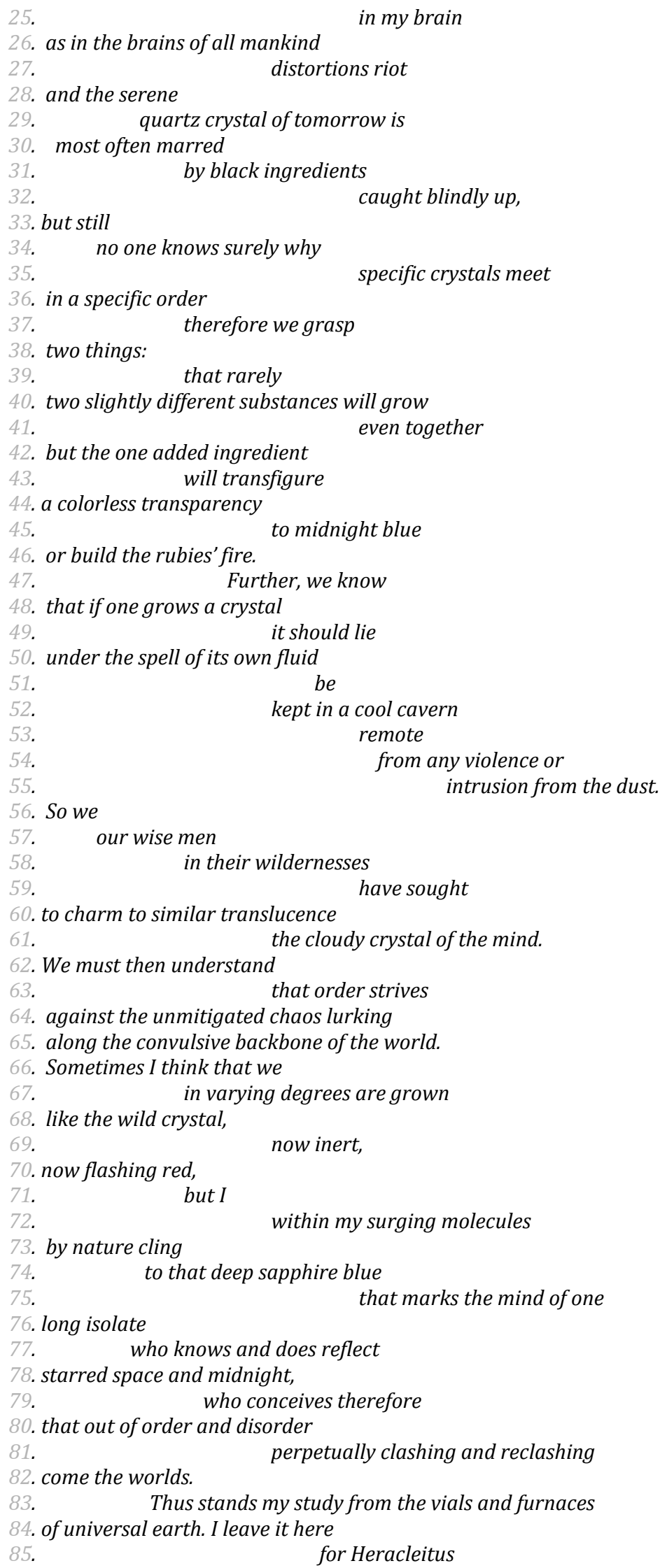


86. if he comes again

87. in the returnings of the Giant Year.

In his poem, Eiseley takes as his theme, the growth of crystals and men. His observation and his phenomenological perspective originate from his "study from the vials and furnaces of universal earth" (line 83), referencing a "teacher frame" in which we understand Eiseley as one observing geological circumstances both from the lab (vials) and the field (furnaces of universal earth) thus establishing for us his viewpoint. We can consider that, as a teacher interacts with students in a formative setting (intellectual growth), Eiseley's theme is built from the observed parallels between his subject matter (crystals) and his audience (students) contributing to the basis for the blend (simile). Remembering the mechanics of our semiotic blending cascade from section 14, let us consider how this system applies to the present poem: 


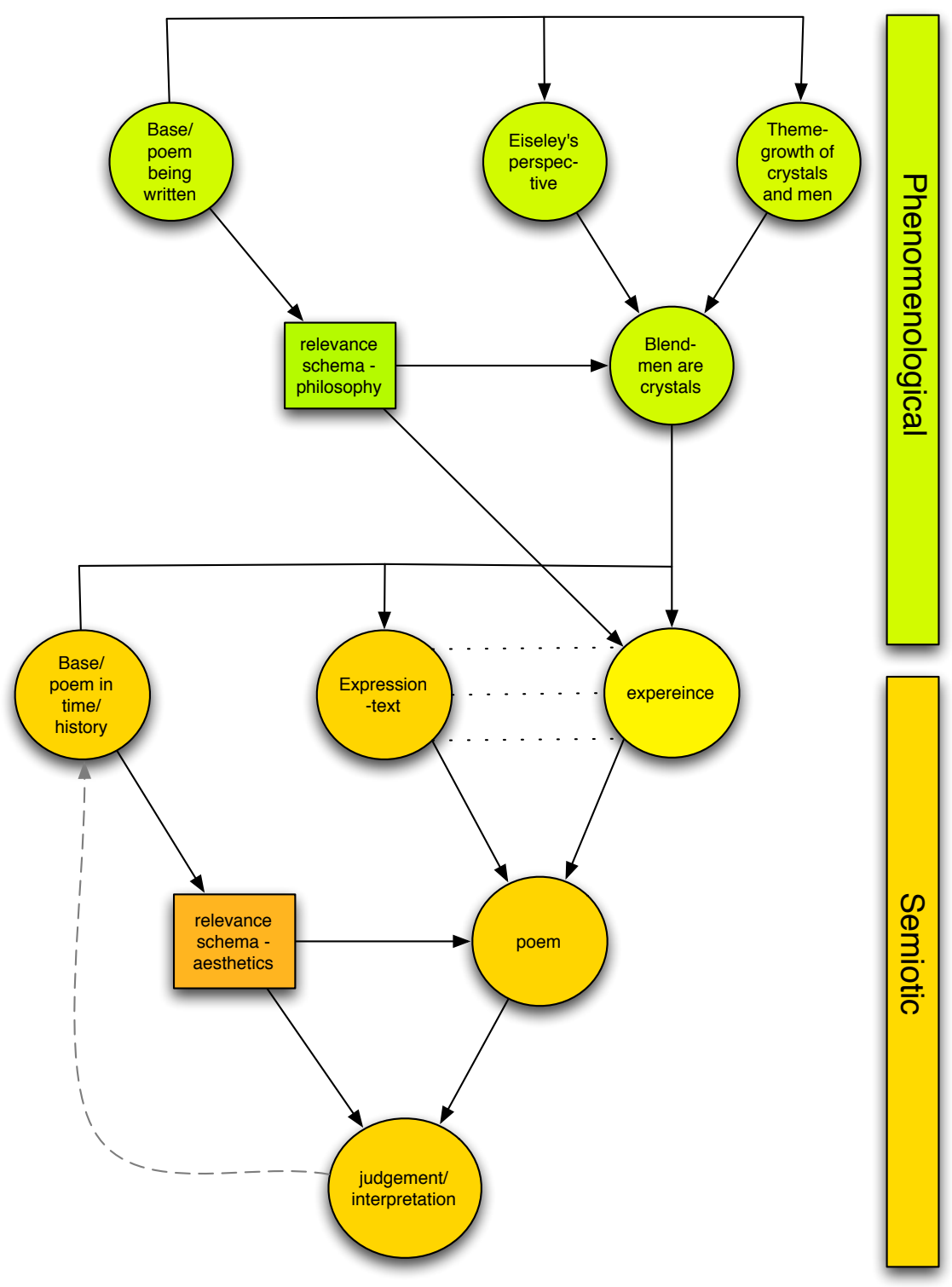

Figure 23 - Semiotic Blending Cascade for the Loren Eiseley poem "Notes of an Alchemist"

As stated in chapter 14, we take as the first two input spaces of the phenomenological layer, Eiselely's theme of the growth of crystals and men, and Eiselely's viewpoint as a nature-science teacher. The base space informs these two inputs and provides the necessary context for this schema. Stabilizing the blend 
(men are crystals) ${ }^{41}$ is our relevance schema; which compounds information from the base space, giving shape to Eiseley's philosophy, which in turn combines with the blend to create his experience space (input space in the semiotic layer). As we learned in section 14, the relevance schema is a dynamic element and takes, in part, the shape of Greimas' semiotic square. In the phenomenological layer of our mapping, this square might look like this:

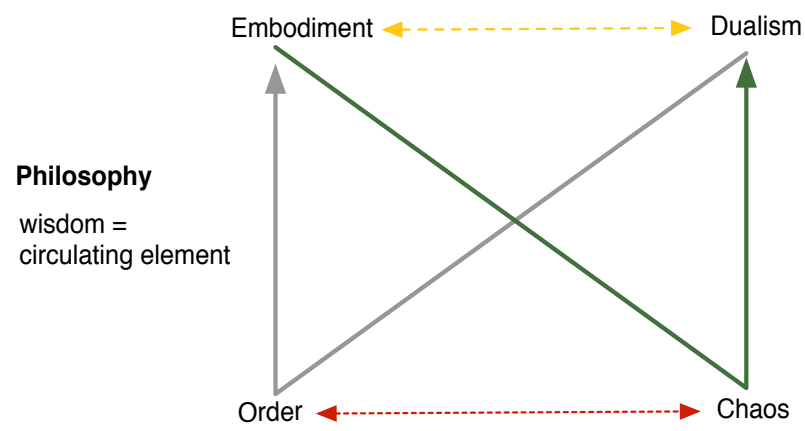

Figure 24 - Semiotic square describing the relevance-making schema in the phenomenological layer of the mapping of Loren Eiseley's poem "Notes of an Alchemist"

The states (dualism and embodiment) and forces (order and chaos) indicated in the mapping above, as defining the network of Eiseley's philosophy, is first indicated in his poem in lines 25-27. Here, Eiseley locates distortion (e.g. chaos) as residing in the brains of all men. In doing do (by locating "distortion" in the brain), the body is not implicated; thus setting up a division between mind and body (dualism). This observation is then contrasted through the use of extended metaphor (in lines 3755) where Eiseley describes how "one added ingredient" will change a formerly transparent crystal to a "midnight blue", referring to the crystals ability to harmoniously co-exist with its occlusion. This transfiguration of the crystal (through

\footnotetext{
41 In this blending cascade, the "blended space" in the phenomenological layer represents a simile, which means that instead of the input spaces containing a source and target as they do in the mapping of a metaphor, the parts of simile are contained in a single space, in this case the blend.
} 
the compounding of two substances) could also be related to the embodiment of man (the two substances being that of the mind and the body) as the sought-after state reflecting an understanding of nature. Eiseley then goes on (in lines 56-65) to introduce the topic of wisdom. He describes "wise men", (who possess distorted brains) as those who have sought "to charm to similar translucence/the cloudy crystal of the mind". In these lines, Eiseley unites the two themes of the poem (crystals and men) by indicating that the mind is a crystal, albeit a "distorted" one, and that wisdom is defined as cloudiness striving towards clarity (e.g. chaos striving towards order). Next, he continues laying out the path for attaining this "wise men" status (in lines 62-65) by stating that "We must understand" that the struggle between order and chaos is ever present in the world ${ }^{42}$ (e.g. nature) and that to strive towards an understanding of this duality, in spite of our distorted brains, is wisdom.

Returning now to Figure 23 (our semiotic blending cascade), remembering that we have already introduced the "experience input", and recognizing that the text as it is reprinted above fills the "expression" input (as it is an expressive instantiation of Eiselely's philosophy); we must be sure to apply knowledge of our own current circumstance (e.g. context) to the base space in the semiotic layer in order to establish, as part of the meaning generation of this work of art, our own experience of it in our own place and time. The relevance schema, which dynamically coalesces features from the base space to stabilize the blend of the experience and expression inputs, provides us with an understanding of Eiseley's aesthetic (of wisdom) as it is

\footnotetext{
42 In lines 63-65 Eiseley describes the world as having a "backbone" - perhaps drawing a connection between
} nature as the spirit inside the body of the "world". 
communicated through his art. Illustrating the relevance schema (of the semiotic layer) we return to our semiotic square:

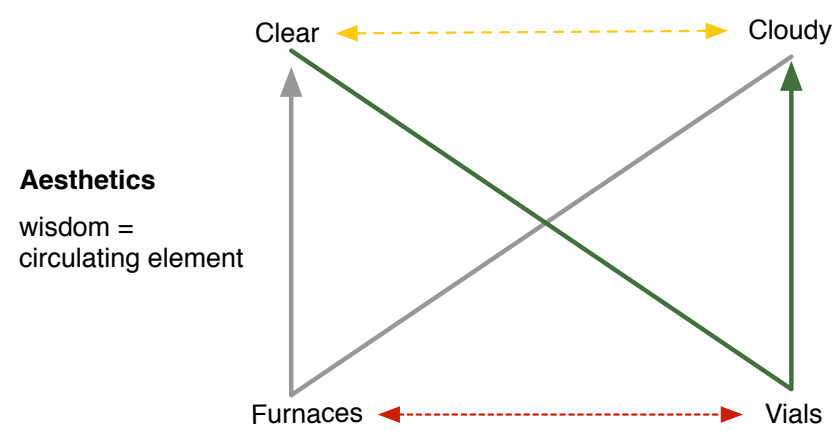

Figure 25 - Semiotic square describing the relevance-making schema in the semiotic layer of the mapping of Loren Eiseley's poem "Notes of an Alchemist"

As we can see from our square, the dynamic oscillating element in the network is again the wisdom of man, but the forces and states have changed. The forces at work (vials and furnaces), describe the opposition between the artificial distortions of man characterized by his substitution of science for nature (vials), and man's observation of natures' processes (furnaces). These forces are then helped or opposed by the states clear and cloudy, which serve to send the dynamic element (wisdom) oscillating through the network. These states, described explicitly by Eiselely in lines 60-61 ("to charm to similar translucence / the cloudy crystal of the mind") correspond to, and define his aesthetic, as nature is clarity, man is cloudy, and the dynamic element wisdom is a process of "perpetually clashing and reclashing" into ones' own cloudiness in search of clarity. 


\section{Reversibility vs. Irreversibility: a Discussion of Temporality in Art}

So far in the present study, we have explored ways of breaking down the elements of theme and narrative within the various modalities of aesthetic expression introduced (poetry, narrative, painting), revealing the consistent presence of dynamic oppositions. The oppositions presented up to this point have remained rooted in the material and are reversible, which means that they can be returned to, recalled, and re-consumed at will. This slow, unraveling experience of an aesthetic object over time, permits and even foregrounds reflection on the present themes, and so supports the audience in their contribution to discourse (conversation in culture). Till now, the forms of aesthetic experience presented fit neatly into our dynamic semiotic square, but there are some circumstances where this might not be so: critical discourse, and the temporal arts. With the following examples, I aim to illustrate these very exceptions using one final (and extreme) variation of our semiotic square graph.

\section{Diegesis: Cy Twombly}

"The vague is alive" - Roland Barthes

In one seminal article on the works (on paper) of expressionist painter $\mathrm{Cy}$ Twombly (born 1928), Roland Barthes ruminates on what might be the "essence" of the artist's work. Questioning whether Twombly's work is based in painting or in 
writing, Barthes identifies the components of the work as being comprised of text, calligraphs, and gesture.

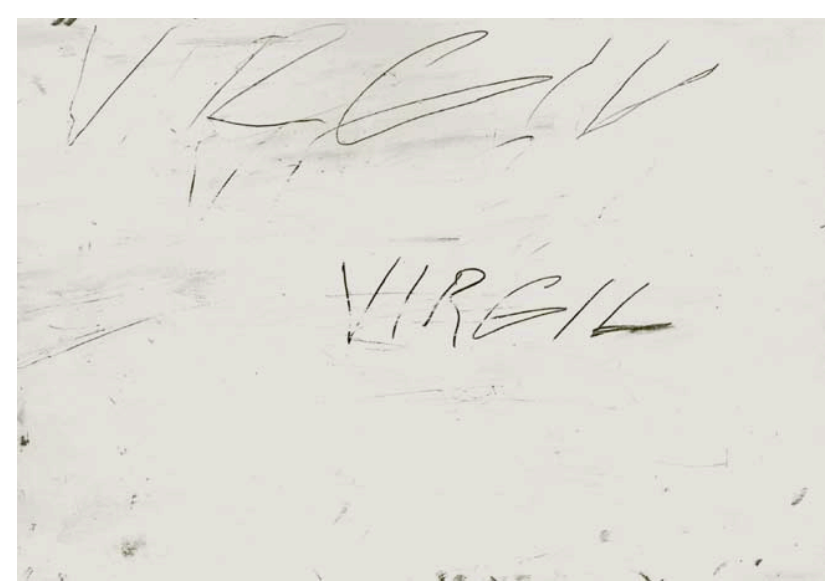

Figure 26 - Work on paper by Cy Twombly entitled “Virgil” (oil, chalk, and crayon on paper). Private collection, Berlin.

While most artists aim to capture some form of experience through the use of technique and medium, thus serving to an audience a work for aesthetic enjoyment, Twombly, it seems, is interested in offering us an artifact of an action. Barthes would agree:

"We are not asked to see, to conceive, to savor the product, but to review, to identify, and, so to speak, to "enjoy" the movement which has ended up here." (Barthes, 164)

So what place does gesture have in the context of material art forms? I would contend that, while all art has, in its making, the celebration of gesture as the delivery of process, Cy Twombly (as do other expressionist painters who focus on mark-making) at times, asks us to foreground the indexical to the detriment of the product (as a focal point). This foregrounding of process serves to implicate gesture 
as the "surplus" of the intentional ${ }^{43}$. But for all its foregrounding, we cannot forsake the object as the genesis of meaning: as the point of reflection. For this, we must again return to Twombly's work as an embodied one straddling the boundaries between life (eros) and death (thanatos), banality and utopia ${ }^{44}$, the reversible and the irreversible, the carnal and temporal. It is here that I introduce our last model:

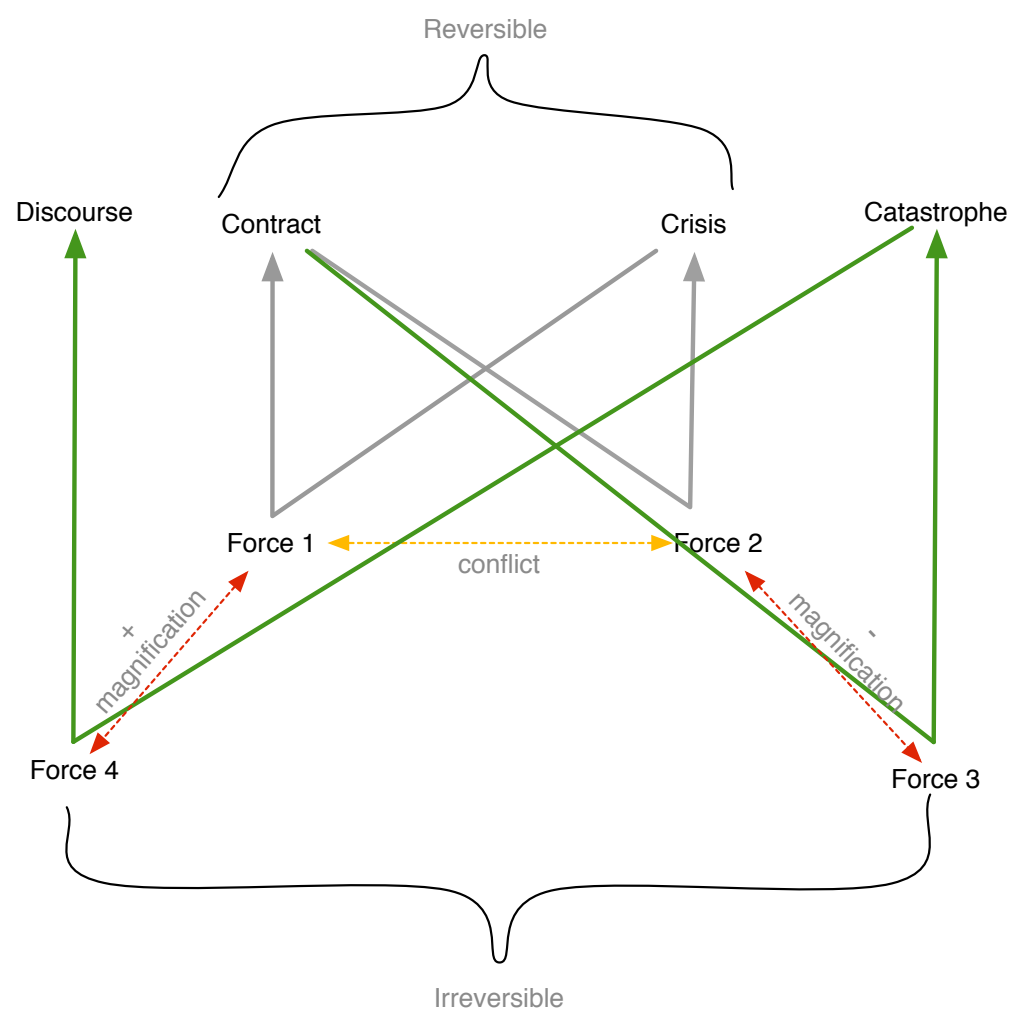

Figure 27 - The (dynamic) semiotic square (grey) extended by the (static) Brandtian Diegesis model (green) indicating the "surplus" of the intentional and showing how an amplification of one of the opposing forces can result in the discontinuation of the dynamic found in the semiotic square.

First let's look at the form of the graph: The grey structure in the center of the network probably looks familiar. It is an unaltered instance of the semiotic square I

\footnotetext{
43 The message which serves to produce information, and the sign which seeks to produce intellection Barthes, Cy Twombly: Works on Paper, page 160

44 Barthes, in his article entitled Cy Twombly: works on paper, describes how art which serves to separate eros (the ife instinct) and thanatos (the death instinct) is banal; and that art which can transcend this resistance of the "human neurosis" and create a "single affect" is the language of utopia.
} 
introduced earlier. What is pertinent to note here, is that the processes and actions that occur within the dynamics of this system are reversible, meaning that the work can be returned to, can be started over. However, there are times when the oscillating element incurs a severe force dynamic event (force 3), which pulls it out of the semiotic square and into the Brandtian diegesis model ${ }^{45}$ resulting in a catastrophe and then a post event discourse. At the discourse point of the model, the formerly oscillating element ceases to be active. What is important to note is that while the semiotic square represents a reversible process, the diegesis model does not; it is irreversible and thus is not wholly dynamic. Let us see this graph as it pertains to Twombly's work:

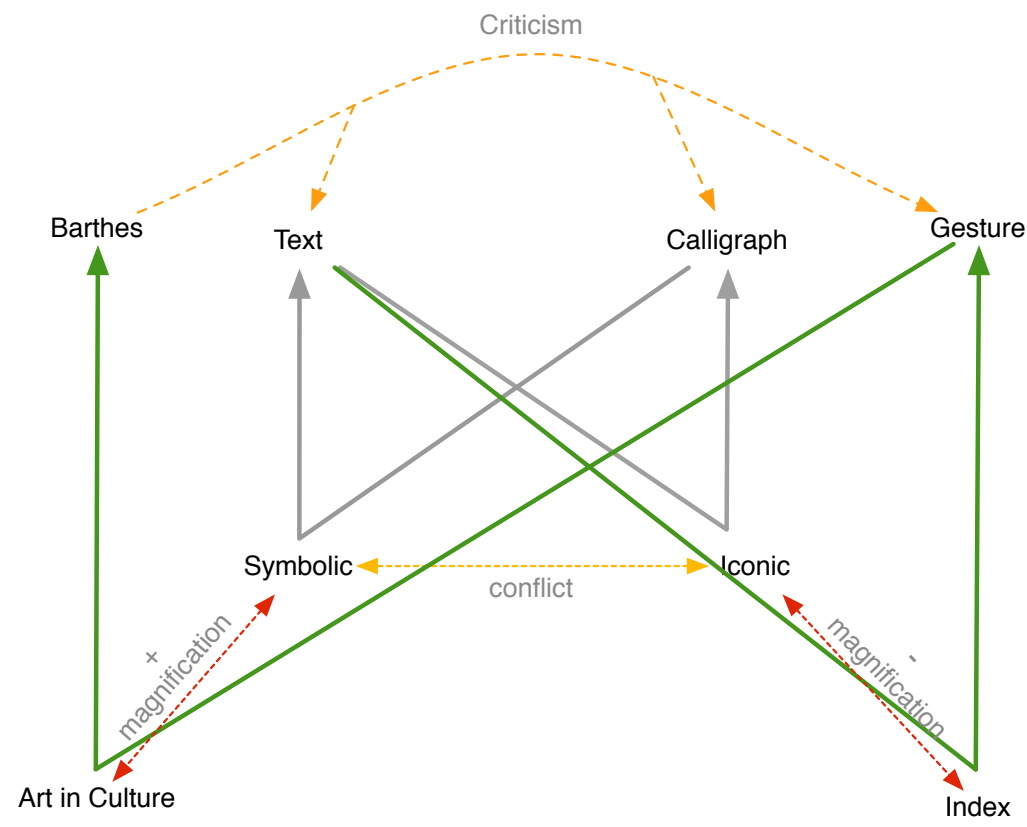

Figure 28 - Semiotic Square and Diegesis Model of Cy Twombly's work entitled "Virgil”.

\footnotetext{
45 This model was first introduced by Per Aage Brandt in his article from 1978 entitled “La diégesis, estructura y proceso de la ficción" (roughly translated as "The diegesis, structures and processes of fiction")
} 
As you can see the graph is the same, but the labeled elements are different. The only omission is that of the labels indicating reversibility and irreversibility, which have been left out only for the sake of visual simplicity. There is, however an additional element that I will explain shortly.

Returning to our image of Twombly's painting entitled Virgil (Figure 26), we can identify easily the labeled element "text" as corresponding to the artist's use of the word "Virgil" as a recognizable (ordered) instance of written language. Next, looking on this work framed contextually as art, we might consider the "style" of his writing, the quality of its line and the amount of emotion we might be able to discern in its application. This perspective creates for us one dilemma Barthes explores in his article; namely, the question of whether Twombly is a writer or a painter. In our graph, this opposing state is labeled "calligraph", the intentional stylization (disordering) of the functional. The oscillation of the element "Twombly's art" then, is pulled through the system by the opposing forces of the symbolic (neg-entropic) and the iconic (entropic) as they relate to or oppose the notions of text and calligraphy. At this point, if I were describing a perpetual dynamic state, one I might illustrate, as I have before, with the semiotic square model, I would stop here. This notwithstanding, our analysis continues.

Returning to Barthes (our narrator), we must recall his assertion that Twombly's work is less about the object than it is about the performance of its creation. This recalls the notion of gesture. While it is true that an element of gesture is present in both the symbolic nature of writing and the iconic nature of painting, in that they both require a kinesthetic action in the intentional creation of their artifact, the 
importance and significance of gesture as it pertains to Twombly's art is in its unintended "surplus"; the moments in his work where he exceeds the iconic, moving instead into a liminal state of intention where he eventually transcends the art object in favor of the fleeting temporal "gesture" as it relates to the performance of creation in time. The liminal state referred to is the amplification of the iconic resulting in the temporary foregrounding of the indexical (Force 3), which leads to the perceptible hyper-state of "gesture" (catastrophe) where Twombly is finally able to exist temporally as a "dancer" of sorts. The aftermath of this transcendence (Art in Culture) serves to again ground the work in the material world while retaining its relationship to time in its connection to history. The magnification of the symbolic, at last, functions to shuttle Twombly's art into the hands of its critic "Barthes".

This brings us to the difference between the "blank" graph used to describe its functionality and the one shown in Figure 28 as the illustration of Cy Twombly's art. In the above graph, the orange dotted line illustrates the reflective nature of criticism, which serves to deconstruct and then analyze the perceived elements of a given work of art.

The analysis of Twombly's art, as it transcends our notion of the dynamic opposition (albeit briefly) on its way to discourse, is still only a transitory accomplice of the temporal; it remains rooted in the materiality of the art object. It is this very reversibility that the critic exploits in his excavation of a work of art as they labor to deconstruct, analyze and define the artist's process, intentionality and significance. But, what of a performance? How might we approach a time-bound work in spite of its apparent irreversibility - our inability to revisit our experience of 
it outside of the realm of memory? Hegel suggests that music (more so than other art forms) is able to free itself from materiality (text):

"I have said already that amongst all the arts music has the maximum possibility of freeing itself from any actual text as well as from the expression of any specific subject matter, with a view to finding satisfaction solely in a self enclosed series of the conjunctions, changes, oppositions, and modulations falling within the purely musical sphere of sounds." (Hegel, 902)

In the above quotation, Hegel separates the experience of music from its notation. The implications of which casts a divide between the audience who is not required to have an understanding of musical notation, and the musician who is held responsible for the articulated and measured expression of a set work according to its prescriptive score ${ }^{46}$. Accordingly, music can be defined as the manifestation of two states: notation and expression. All composed music, then, oscillates between these states driven by the opposing forces of the symbolic (adherence to prescription) and the acoustic (expression through sound). Again, the pattern of order (neg-entropy) and disorder (entropy) emerge as the opposition between the imposed ideal of symbolic notation and the entropic tendency of expression, which seeks to erode the symbolic order in pursuit of a transcendent and temporally realized work of art. If we were to illustrate this as before with our semiotic square model, it would look like this:

\footnotetext{
46 To assume the irrelevance of musical notation to the audience is to either assume a certain amount of illiteracy, or to intentionally remove the frame of reference that a musical score provides. This is the particular practice of classical (and often pop) music, but is not the convention across all genres. Commonly, amongst performances of the avant-garde (or the experimental), the score is provided to the audience as a necessary guide to the understanding and appreciation of the music. Also, in traditional jazz, the theme or harmonic structure of the chorus, is the 'score'. This score is acknowledged but abandoned in "free jazz" where the acoustic is foregrounded (soloing) and the theme is usually not returned to.
} 


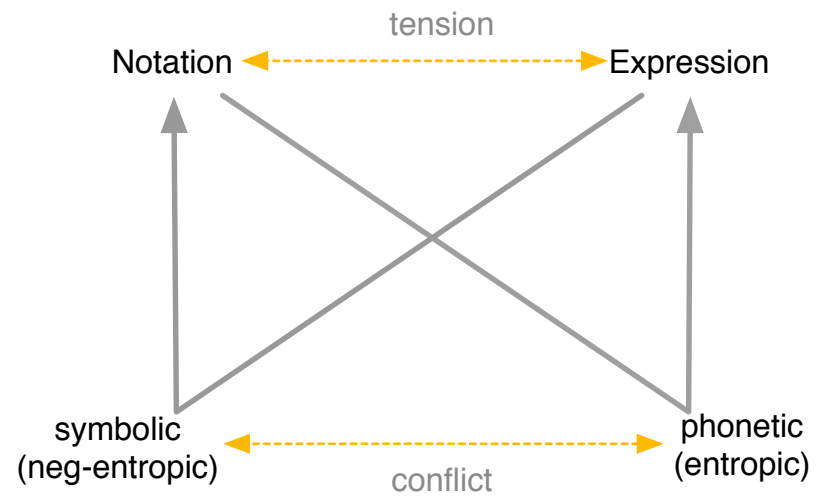

Figure 29 - semiotic square model of music

The significance of this structural dynamic is in how it relates to and parallels the human experience. Art, as the product of an intentional and culturally framed decision-making process conducted by human beings as a reaction to, or expression of their experience, has so far proven to mirror the "deep structure" of human embodiment defined here as the opposition of entropic and neg-entropic forces. However, the reversibility of this recursive dynamic structure largely depends either on the materiality of the notation, or the audience's control over the circumstance in which the music is experienced - namely that of time and sequence. In order for music to truly transcend the materiality of art, as Hegel suggests, the audience must relinquish control over all aspects of the performance, and have little if any access to any specifically prescriptive notation such as that of a musical score.

In order to explore the structure of the musical experience further, and to analyze the importance and significance of notation on the overall aesthetic, I will now look at a modern work by English avant-garde composer Cornelius Cardew 
(1936 - 1981) ${ }^{47}$ entitled Paragraph 7; a piece taken from the album "The Great Learning" from 197148. The score for Paragraph 7 looks like this:

\author{
Paragraph 7 \\ 1/2 page \\ for any number of untrained voices \\ Duration about 90 minutes \\ Composed 08.04.69
}

sing 8 IF

sing 5 THE ROOT

sing 13 (f3) BE IN CONFUSION

sing 6 NOTHING

sing 5 (f1) WILL

sing $8 B E$

sing 8 WELL

sing 7 GOVERNED

hum 7

sing 8 THE SOLID

sing 8 CANNOT BE

sing 9 (f2) SWEPT AWAY

sing $8 \mathrm{AS}$

sing 17 (f1) TRIVIAL

sing 6 AND

sing 8 NOR

sing 8 CAN

sing 17 (f1) TRASH

sing 8 BE ESTABLISHED AS

sing 9 (f2) SOLID

sing 5 (f1) IT JUST

sing 4 DOES NOT

sing 6 HAPPEN

hum 3 (f2)

speak 1 MISTAKE NOR CLIFF FOR MORASS AND TREACHEROUS BRAMBLE

Figure 30 - Score for Cornelius Cardew's The Great Learning from 1971, taken from paragraph 7 of the Confucian text of the same name. Text translated into English by Ezra Pound.

47 Cardew served as an assistant to Karlheinz Stockhausen from 1958 to 1960 where he likely learned his skillful mastery over the serialist languages often used by Stockhausen.

48 The piece was first composed in 1969, but was not performed formally until 1971. 
From the first glance it is clear that the above score lacks the usual musical notation (e.g. notes on a staph) and instead has replaced it with that of an unconventional and vague methodology ${ }^{49}$. Each line is to be sung the indicated number of times, (sing 8, sing 5) and is to be sustained for the duration of one breath. The notation (f1) means that the line should be repeated forte ${ }^{50}$ at least one time out of the number of repetitions otherwise indicated. On closer inspection, the differences between it and classical forms of notation go further: the performers of this piece of music are explicitly required to be a mixture of trained and untrained musicians, there is no standard of time, and no particular instructions related to pitch. Some might suggest that because the notation (in conjunction with a set of untrained musicians) cannot guarantee the reproducibility of the piece, Cardew has given us a score at all. Brian Eno, an English musician, composer, record producer, and music theorist explains that this is a distinction between classical and Avantgarde (or experimental) tendencies:

I hope to show that an experimental composition aims to set in motion a system or organism that will generate unique (that is, not necessarily repeatable) outputs, but that, at the same time, seeks to limit the range of these outputs. This is a tendency toward a "class of goals" rather than a particular goal, and it is distinct from the "goalless behavior" (indeterminacy) idea that gained currency in the 1960s.

With the preceding quotation, Eno is suggesting that the distinction between the classical and experimental genres is that classical music strives towards a "particular goal" (neg-entropic) whereas experimental music endeavors towards a "class of goals"(entropic). So, while a trained classical musician might listen to a

\footnotetext{
49 The group of trained and untrained singers is to sing the line indicated on any 1 note they wish, so long as they do not repeat the note for two lines in a row. The duration of each line is to last for one breath. This length of time will (and is supposed to) vary amongst the different singers.

50 The term forte means to "play loud"
} 
piece of music, refer back to its notation, (as one might linger over the text of a narrative) and be able to access some level of its aesthetic cache, the experimental musician, whose work might never sound the same twice, would have no such aesthetic access through notation beyond that of the score itself ${ }^{51}$. This would suggest that a piece of music like Paragraph 7 has a stronger connection to the temporal than that of a conventional classical work. Eno agrees:

The composer [of experimental music], instead of ignoring or subduing the variety generated in performance, has constructed the piece so that this variety is really the substance of the music.

The weaker the connection between the experience of a piece of music and that of its notation, the more effectively it is able to fully transcend the material (notation, the visual performance, the recording) in favor of a truly temporal state (the fleeting decay of sound over time).

But, there is more to a piece of music's ability to transcend the material than just its relationship to its score; there seems to be a further distinction between the practice and the performance frames as they relate to the live production of music. When a group of musicians (or a single musician) is rehearsing (thus denoting occupation of the practice frame) and a mistake is made, it is possible for the group to stop and review the section containing the difficulty. In a performance however, the ability to reverse the musical experience is not observed as an optional component of the performance frame (according to situational constraints or

\footnotetext{
51 It is prevalent amongst avant-garde musicians, to experiment with graphical systems of notation. While these types of scores generally suggest a more or less loose set of guidelines for those interpreting them, they usually leave room for subjective interpretation. Many of these kinds of scores end up being visually interesting and thus examples of aesthetic objects themselves.
} 
contextual conventions). Therefore, the experience of a formal musical performance suggests a higher level of temporality than that of a rehearsal; and when this performance is of an experimental work, is less constrained by the text (notation) related to it. Let us now explore the structural dynamics of Cardew's transcendence of the material into the temporal by returning to our Brandtian diegesis graph:

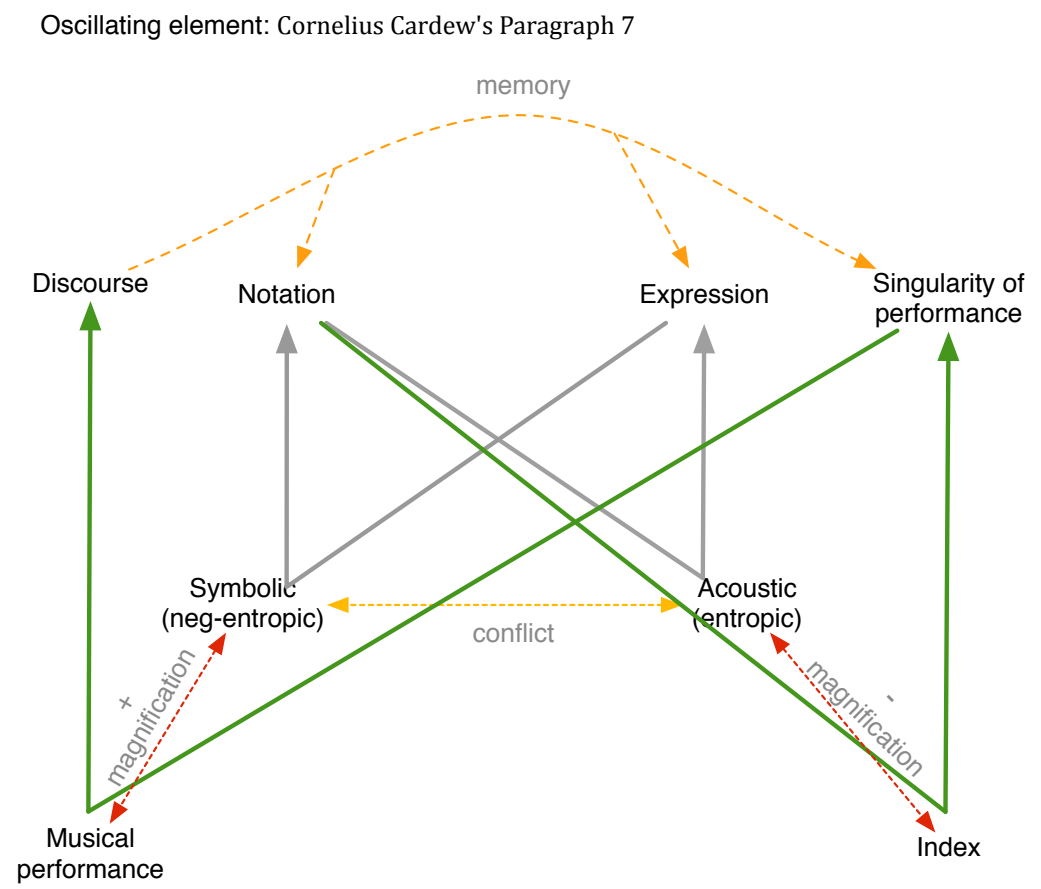

Figure 31 - Semiotic Square and Diegesis Model of the musical piece by Cornelius Cardew's entitled Paragraph 7

In Cardew's Paragraph 7, the loose notation, or score, is expressed freely by the singers involved in its performance. This oscillation between the symbolic (negentropic) and the acoustic (entropic) define the semiotic square. The intentionality of the music however, asks us to foreground the notation - as the significance of the piece of music is defined by its arrangement and not the expression of its score. This 
foregrounding serves to pull the dynamic state of notation into a hyper-state here named the indexical. In the application of the term as used here, the index refers to the evidence of a sign that indicates some state of affairs or being. In this case, the notation or score of Paragraph 7 is extended and magnified through its lack of specificity and foregrounding and serves to highlight for the audience, the singularity of the experience. In other words, while the score of Paragraph 7 serves to create an index of the circumstance (acoustics, selection of singers, parameters) of the performance, the intentional and entropic drive towards a "class of goals" function to underscore the singularity of the experience. This singularity, and thus irreversibility of the performance, effectively transcends the dynamic and recursive system, instead focusing the experience on the purely temporal (labeled above as "musical performance"). The temporal experience of music then, is transported into discourse only by the vehicle of subjective memory (the reflection of which is indicated by the orange arrows). It is this inability to return to the performance (by going back in time) that characterizes Cardew's Paragraph 7 as a dissipating memory within the framework of an individually experienced history and engagement with culture (dialogue).

These last two examples of artistic expression continue to strongly articulate the structural presence of the force dynamic opposition between order (neg-entropic) and disorder (entropic) as embodied through the dynamic states identified in the semiotic squares. However, what is interesting is that the difference between reversibility and irreversibility as made distinct though the differing functions of the semiotic square and diegesis models, seem to implicate the original difficulty that 
Arnheim had in reconciling his theory of art with that of his understanding of the physics of entropy. The solution to this disparity lies (I think), in the difference between the semiotic square model as illustrative of an open system - one that continuously oscillates between states, and the diegesis model as representative of a closed system - one that moves from one static state to that of an end state.

A case can be made that the end state of a diegesis model (being discourse) is not a firm representation of a "closed" system (in that is takes place in culture), but I would contest that the memory of the experience referenced, residing if only partly (as in Twombly) in the temporal, would degrade over time resulting in the "dissipation" of the productivity of the dialogue. This end state, reached through the removal or transcendence of the materiality of the aesthetic experience brings us very close to the original (and unaltered) definition of entropy as "a tendency (in a closed system) towards the dissipation of useful energy".

\section{Conclusion}

\section{"All art is self-portraiture."}

- Oscar Wilde

As I have shown, the dynamic opposition of order and disorder is consistently represented throughout the different artistic modalities explored. Regardless of genre, medium or subject matter, the structure of human embodiment, expressed though the dynamic opposition of body and mind is ever-present in any intentional work of art, and thus contributes to the cognitive framework on which we build our 
understanding of aesthetic experience. This deep structure, first approached by Rudolf Arnheim as relating to the opposition of order and disorder within a system as explained by the $2^{\text {nd }}$ law of thermodynamics, indeed exists, and serves to provide insight into an essential component of what it means to be human.

Art, like language, is symbolic and we as a symbolic species are unique in the world for being able to understand, create and share these symbols with each other. Exploring the depths of the aesthetic experience, then, is not trivial. To look for signs of some unifying principle by which we recognize something as "aesthetic" is an investigation into critical human processes. It goes beyond the notion of the "new" in how it pertains to creativity (the act of creating) and instead inquires into the underlying cognitive structure of the aesthetic experience. The art world has long been interested in the pursuit of universal beauty and while this study has not been interested in supplying parameters for subjective evaluation, I believe some glimpse of a special sensibility relating to the aesthetic has been identified in the force dynamic opposition of order and disorder.

Oscar Wilde's character, Basil Hallward in The Picture of Dorian Grey states, "All art is self-portraiture". While the art world has taken many views of this assertion, applying it to notions of self-reflexivity, and a forensic view of art, I have shown that the significance of this statement is derived not from its reference to a work of art as a reflection of the intentional viewpoint or preference of the artist, but rather in how it speaks on a deep level of the innately human experience of phenomenological embodiment. Just as we are an embodied and symbolic species, so too does our art reflect this dynamic. It is representative of and mirror to our experience and 
perspective as human beings, and so is structured like that of our awareness; body and mind, emotion and expression, tangible and intangible, order and disorder.

\section{Implications for Further Research}

Some suggestions for further research to extend and further elucidate the present topic would be to explore the psychology of the embodied human condition and how it relates to abstraction as it is referred to by neurologist Semir Zeki, in his article entitled Neural Concept Formation \& Art: Dante, Michelangelo, Wagner (Zeki, 2002). In this article, Zeki defines art as:

"A by-product of this abstracting, concept-forming, knowledge-acquiring system of the brain and can only be understood biologically in that context."

I would be interested to know if embodiment (as a force dynamic relationship between order and disorder) is or can be recognized in the same way as spatial relationships (like the notion of verticality). If the brain constantly processes information by first looking for general consistencies as Zeki suggests, and all art is built upon a structure defined by human embodiment, it is conceivable that there could be a neurological basis for aesthetics.

This in turn leads to another direction for this line of inquiry: a more in-depth investigation into the temporal arts. If we are to understand the aesthetic experience as the force dynamic opposition of order and disorder, and that experience is distributed in time; how then, is the dynamic process of perception situated in time in relation to a time-based (temporal) work? Mark Twain wrote: 
"Life does not consist mainly -- or even largely -- of facts and happenings. It consists mainly of the storm of thoughts that is forever blowing through one's head."

From this perspective, we might conceive of our ephemeral aesthetic experience as parallel to the fading decay of sensation and memory. That perhaps we live in different temporalities and that through the miracle of converging conditions, we perceive a singularity unique to us and our window on the world. In this way, we are all artists; we are all painters of a unique narrative, and because we are human and can make, perceive, and understand art. The pursuit of a greater knowledge of the cognitive underpinnings of the aesthetic experience is central to our gaining an understanding of what it means to be human. I do not end but here with this quote from a teacher and mentor, Mark Turner:

Artists are cognitive researchers in the wild. It would perhaps be a scientific advantage if cognitive researchers were also artists themselves. Anyway, the study of the psychology of aesthetic sensibility, or "psycho-aesthetics," is a dimension of cognitive science that future research will need to look closely into. (Turner, 183) 


\section{Bibliography}

Arnheim, Rudolf. "A Plea for Visual Thinking." Critical Inquiry 6.3 (1980): 489-497. Arnheim, Rudolf and Arthur Karp. "From Contrast to Assimilation: In Art and in the Eye." Leonardo 8.3 (1975): 270-271.

Arnheim, Rudolf. Art and Visual Perception: A Psychology Of The Creative Eye. Los Angeles: University of California Press, 1974.

-. Entropy and Art: an Essay on Disorder and Order. Los Angeles: University of California Press, Berkeley, 1971.

—. "From Chaos to Wholeness." The Journal of Aesthetics Art Criticism 54.2 (1996): 117-120.

—. "Gestalt Misapplied (Letter to the editors)." Contemporary Psychology (CP) 19.7 (1974): 570.

—. "On Order, Simplicity and Entropy." Leonardo 7.2 (1974): 139-141.

—. "The Priority of Expression." The Journal of Aesthetics and Art Criticism 8.2 (1949): 106-109.

—. "Why Words Are Needed." Journal of Aesthetic Education 32.2 (1998): 21-25.

Balzac, Honoré De. The Unknown Masterpiece. New York: New York Review Books, 2001.

Baudelaire, Charles. Les Fleurs Du Mal. Ed. Richard Howard. Boston: David R. Godine, 1982.

Ben-Naim, Arieh. Entropy Demystified: The Second Law Reduced to Plain Common Sense (with seven simulated games). New Jersey: World Scientific Publishing Co.

Pte. Ltd., 2008.

Brandt, Per Aage and Line Brandt. "Cognitive Poetics and Imagery." European Journal of English Studies 9.2 (2005): 117-130.

Brandt, Per Aage. "Diegética y fantasmática. Dos dimensiones fundamentales del objeto semiótico." Semiosis 25.uly-December (1990): 81-102.

-. Spaces, Domains, and Meaning: Essays in Cognitive Semiotics, European Semiotics Series No. 4. New York: Peter Lang, 2004.

—. "The world. A view from within (working paper)." (2010).

Brasseur, Lee Ellen. "Visual Thinking in the English Department." Journal of Aesthetic Education 27.4 (1993): 129-141.

Fauconnier, Gilles. Mappings in Thought and Language. Cambridge: Cambridge University Press, 1997.

-. Mental Spaces: Aspects of meaning construction in natural language. New York: Cambridge University Press, 1885.

Freund, John. "Entropy and Composition." College English 41.5 (1980): 493-516.

Gombrich, E.H. Art and Illusion: A study in the psychology of pictorial

representation. New York: Phaidon Press, 2002.

Greimas, A. J. and J. Courtes. Semiotics and Language: An Analytical Dictionary.

Bloomington: Indiana University Press, 1892. 
Case Western Reserve University

Greimas, A.J. Structural Semantics: An Attempt at a Method. Lincoln: University of Nebraska Press, 1983.

Harrison, Charles, Paul Wood and Jason Gaiger, Art in Theory 1815-1900: An

Anthology of Changing Ideas. Malden: Blackwell Publishing, n.d.

Hawkes, Terence. Structuralism and Semiotics. New York: Routledge, 2003.

Hoare, Michael, et al. "On Entropy and Art (Continued)." Leonardo 6.3 (1973): 282284.

Jones, Peter Lloyd. "Some Thoughts on Rudolf Arnheim's Book "Entropy and Art"." Leonardo 6.1 (1973): 29-35.

Krauss, Rosalind. "Notes on the Index: Seventies Art in America." October 3 (1977): 68-81.

—. "Notes on the Index: Seventies Art in America. Part 2." October 4 (1977): 58-67. Kristeller, Oskar Paul. "The Modern System of the Arts: A Study in the History of Aesthetics Part 1." Journal of the History of Ideas 12.4 (1951): 496-527.

Landsberg, Peter T. Seeking Ultimates: An Intuitive Guide To Physics. IOP Publishing Ltd., 2000.

Martin, Bronwen and Felizitas Ringham. Dictionary of Semiotics. New York:

CASSELL, 2000.

Merleau-Ponty, Maurice. Phenomenology of Perception. Trans. Colin Smith. New

York: Routledge Classics, 2002.

-. The Merleau-Ponty Aesthetics Reader: Philosophy and Painting. Ed. Galen A.

Johnson. Trans. Michael B. Smith. Evanston: Northwestern University Press, 1993.

Stern, August. Quantum Theoretic Machines: What Is Thought From The Point of

View Of Physics. Amsterdam: Elsevier Science B.V., 2000.

Stevens, Wallace. Parts of a World. New York: AA Knopf, 1942.

Turner, Mark. The Artful Mind: Cognitive Science and the Riddle of Human

Creativity. New York: Oxford University Press, 2006.

Wessell Jr., Leonard P. "Alexander Baumgarten's Contribution to the Development of Aesthetics." The Journal of Aestheticsa and Art Criticism 30.3 (1972): 333-342. 